\title{
Lücken im kriminalpolitischen Diskurs
}

- Zu den Gesetzentwürfen zur Verbesserung des Schutzes der Intimsphäre ${ }^{1}$ -

\section{Einleitung}

Nachdem sich die Sicherheitspolitiker und ihnen folgend die Gesetzgeber in Bund und Ländern in Kämpfe gegen globale Bedrohungen ${ }^{2}$ und die Gefährlichsten der Gefährlichen $^{3}$ verstrickt haben, melden sich nun offenbar die Rechtspolitiker in den Gefilden des nationalen Kernstrafrechts zurück: Der strafrechtliche Schutz des »persönlichen Lebens- und Geheimbereichs « (15. Abschnitt des StGB-BT) soll »verbessert« werden. Beabsichtigt ist aber nicht etwa eine durchaus erwägenswerte Gesamtreform dieses Deliktsbereichs ${ }^{4}$ unter Einbeziehung nebenstrafrechtlicher Ergänzungen (s.u. III. 3.), sondern abermals ${ }^{5}$ das Stopfen einer vermeintlichen Lücke.

1 Überarbeitete Fassung einer Stellungnahme, die der Verf. am 24.9.2003 vor dem Rechtsausschuss des Bundestages im Rahmen einer öffentlichen Anhörung (s.u. Anm.15) abgegeben hat.

$2 \mathrm{Zu}$ den sicherheitspolitischen und gesetzgeberischen Hyperaktivitäten nach dem 11.9.2001 im Überblick Roggan, Handbuch zum Recht der Inneren Sicherheit, Bonn 2003 (insb. S.169 ff.), vgl. auch Pollähne, ansprüche 1/2003, 7 ff.

3 Neben den neuerlichen Verschärfungen des Sexualstrafrechts (BT-Drs 15/1311) fällt besonders ins Auge, mit welcher sicherheitspolitischen und ahistorischen Selbstverständlichkeit quer durch nahezu alle politischen Lager die Sicherungsverwahrung zum Mittel der Tagespolitik in Bund und Ländern anvanciert ist, vgl. Rzepka, R\&P 2003, 127 ff., 191 ff. m.w.N.

4 Zur Begründung dieses seinerzeit »vorbildlosen« Abschnitts vgl. E'62 S.326 ff. (vgl. Schwalm ZStW 74, 488 ff.), wo bereits auf »erhöhte Gefahren« für den Schutz der Privatsphäre u.a. durch »Einrichtungen der modernen Massengesellschaft, Sensationsgier, rücksichtsloses Gewinnstreben sowie die Entwicklung und laufende Vervollkommnung technischer Mittel « verwiesen wurde (aaO S.326, ähnlich AE-StGB-BT 1971, 29,33); grundlegend dazu Kienapfel, Privatsphäre und Strafrecht, Frankfurt/M. 1969; Arzt, Der strafrechtliche Schutz der Intimsphäre, Tübingen 1970 und Peglau, Der Schutz des allgemeinen Persönlichkeitsrechts durch das Strafrecht, Frankfurt/M. et al 1997; vgl. auch Blei JA 1974, 601 ff., Lenckner FS Baumann 1992, 139 f. und Überblick bei Schmitz JA 1995, 31 f. - zur rechtspolitischen Diskussion (u.a. über sog. Indiskretionsdelikte) Rogall FS Hirsch 1999, 665 ff. und 692 sowie bereits Schünemann ZStW 1978, 11 ff., 34 ff. und Henkel DJT 42 D 63 ff.; zur rechtshistorischen Entwicklung Austermühle, Zur Entstehung und Entwicklung eines persönlichen Geheimsphärenschutzes ..., Berlin 2002 (insb. S.155 ff. und 184 ff.).

5 Exempl. der dem 25. StrÄndG vom 20.8.1990 (BGBl.I, 1764, dazu ZRP 1991, 169 und JuS 1991, 169) zugrundeliegende Entwurf der damaligen CDU/CSU/FDP-Koalition vom 15.3.1990 (BT-Drs. 11/6714 und 7414) sowie die dem vorangegangenen Entwürfe (BTDrs 8/2396 der CDU/CSU und 8/2545, 9/719 und 10/1618 des Bundesrates), krit. dazu Lenckner FS Baumann 1992, 135 ff., vgl. auch Schilling JZ 1980, 7 ff. 
Kaum hatte der Bundesbeauftragte für den Datenschutz in seinem 18. Tätigkeitsbericht an den Gesetzgeber »appelliert«, im StGB »eine Regelung für die Fälle zu schaffen, in denen mittels Bildaufnahme bzw. -veröffentlichung unbefugt in den Kernbereich der Privatsphäre und in die Intimsphäre eingegriffen wird « ${ }^{6}$, entbrannten parlamentarische Aktivitäten. Die FDP fragte schon wenige Wochen später schriftlich im BMJ an, inwieweit die Bundesregierung prüfe, ob auch »die heimliche Beobachtung mittels Videokamera etc. zukünftig strafbar sein soll« (BT-Drs. 14/6117 S.6), und erhielt zur Antwort den Hinweis darauf, es werde in der Tat bereits eine Vorschrift vorbereitet, »wonach unbefugte Bildaufnahmen und die unbefugte Beobachtung mit einem technischen Gerät (z.B. einer Videokamera) mit Strafe bedroht werden soll. Diese Vorschrift soll gemeinsam mit anderen Änderungen in einen Gesetzentwurf zur weiteren Überarbeitung des Besonderen Teils des Strafgesetzbuches aufgenommen werden« $(\mathrm{aaO})$. Zwar wurde über diesen Gesetzentwurf zwischenzeitlich als »Eckpunktepapier « berichtet ${ }^{7}$, er hat das BMJ aber offiziell nie verlassen - später ging offenbar die 9-11-Welle darüber ebenso hinweg, wie über einen bereits Ende 2001 vorgelegten eigenen Gesetzentwurf der FDP »zum verbesserten Schutz der Intimsphäre $\ll^{8}$ Dieser wurde nahezu unverändert Anfang 2003 erneut eingebracht ${ }^{9}$ und nicht nur von der Parteikollegin an der Spitze des Baden-Württembergischen Justizministeriums ${ }^{10}$ sondern - ebenfalls nahezu unverändert ${ }^{11}$ - unter demselben Datum von der CDU/CSU übernommen ${ }^{12}$, während die Bundesregierung weiterhin schwieg. Den vorläufigen Schlusspunkt setzte Bayern mit der Vorlage eines (wirklich) eigenen Entwurfs, der am 10.9.2003 im Rechtsausschuss des Bundesrates eingebracht wurde und den Entwurf aus Baden-Württemberg weitgehend ablöste. ${ }^{13}$ All diese Entwürfe waren Gegenstand einer öffentlichen Anhörung des federführenden ${ }^{14}$ BT-Rechtsausschusses

6 BT-Drs 14/5555 S.22 Ziff.1.6, vgl. Schnorr/Wissing ZRP 2001, 279 sowie Manzewski und Beck BT-PlenProt 14/221 S.22005 ff.

7 ZRP 2001, 391 und Manzewski BT-PlenProt 14/221 S.22005 f., vgl. dazu Pollähne R\&P 2001, 223.

8 BT-Drs 14/7193 v. 18.10.2001, am 28.2.2002 an die Ausschüsse überwiesen (PlenProt 14/ 221 S.21991 f.), aber nicht mehr abschließend beraten (vgl. die Positionen der BT-Fraktionen dazu aaO S.22005 ff.)

9 BT-Drs 15/361 v. 29.1.2003 (nachfolgend E-FDP, soweit nicht unmittelbar aus der Drs. zitiert wird).

10 BR-Drs 164/03 v. 11.3.2003 (E-BaWü), der allerdings später durch den E-Bayern ersetzt wurde (s.u.); vgl. zum E-BaWü auch das Rechtsgespräch mit Corinna Werwigk-Hertneck in ZRP 2003, $293 \mathrm{ff}$.

11 Ein bemerkenswerter Vorgang koalitionärer Opposition: Die Übernahme geht bis zur Kopie von Textbausteinen und Fehlern.

12 BT-Drs $15 / 533$ v. 11.3.2003 (nachfolgend E-Union, soweit nicht unmittelbar aus der Drs. zitiert wird).

13 Dass der bayrische Entwurf (nachfolgend E-Bayern, vgl. dazu Detjen ZRP 2003, 222) nunmehr als Entwurf Baden-Württembergs gehandelt wird (BR-Drs 164/1/03 vom 16.9.2003 als Vorlage für die 791. BR-Sitzung am 26.9.2003 = BT-RA-Drs 15 (6) 41 vom 17.9.2003, vgl. NJW 44/2003 S.VI), erleichtert nicht gerade den Versuch, sich einen Überblick über die vorliegenden Gesetzentwürfe zu verschaffen; der E-Bayern wurde jedenfalls nachträglich zum Gegenstand der Anhörung des BT-Rechtsausschusses gemacht (s.u. Anm.15). 
am 24.9.2003 ${ }^{15}$, an deren Rand inoffiziell zu erfahren war, dass sich die rot-grüne Koalition offenbar auf einen eigenen Entwurf verständigen und tendenziell dem EBayern folgen werde. ${ }^{16}$

Mit einer Änderung der $\S \S 201$ ff. StGB, genauer: mit der Füllung einer vermeintlichen Lücke ist also auf die eine oder andere Weise in absehbarer Zeit zu rechnen. Grund genug, sich mit den vorgelegten Entwürfen etwas eingehender zu befassen.

\section{Lücken}

Die vorliegenden Gesetzentwürfe »zum verbesserten Schutz der Intim- bzw. Privatsphäre « sind Produkte eines kriminalpolitischen »Lücken«-Diskurses, der sich bei näherer Betrachtung nicht nur als lückenhafter Diskurs erweist, sondern auch Lücken in den Begründungen aufweist und die mehr als lückenhafte Verfolgung solcher Delikte ignoriert.

\section{Lücken-Diskurs}

Der Verweis auf (mindestens) eine Lücke im strafrechtlichen Schutz »des persönlichen Lebens- und Geheimbereichs « ist ebenso wenig neu (s.o.) wie die damit - vermeintlich zwangsläufig - korrespondierende Forderung nach deren Füllung. ${ }^{17} \mathrm{Neu}$ ist aber auch nicht die Erkenntnis, dass weder das Strafrecht insgesamt noch speziell der in Frage stehende 15.Abschnitt des StGB-BT in der Lage sind, einen lückenlosen Schutz (hier: gegen Verletzungen der Intim- und Privatsphäre) zu gewährleisten. ${ }^{18}$ Solange eine reale oder vermeintliche »Lücke « im strafrechtlichen Rechtsgüterschutz nicht lediglich auf einem gesetzgeberischen Versehen beruht ${ }^{19}$, impliziert der bloße Verweis auf eine solche weder die Notwendigkeit ihrer Füllung noch macht er die

14 Mitberatend sind - naheliegend - der Innenausschuss und - nicht ganz so naheliegend der Ausschuss für Familie, Senioren, Frauen und Jugend zu Stellungnahmen aufgefordert, die jedoch im November 2003 noch nicht vorlagen.

15 Die Anhörung war ursprünglich für Mai 2003 geplant, dann aber wegen der anstehenden BR-Beratungen in den Herbst verschoben (vgl. Detjen ZRP 2003, 222); teilgenommen haben: Prof. Dr. Carl-Eugen Eberle als Justiziar des ZDF, Prof. Dr. Dr. Kristian Kühl von der Universität Tübingen, Prof. Dr. Heinz Schöch von der Universität München, die Leitende Oberstaatsanwältin Dr. Christine Hügel aus Konstanz, der Oberstaatsanwalt beim BGH Dr. Ulrich Franke und Dr. Roland Helgerth von der Generalstaatsanwaltschaft beim BayObLG sowie Burkhard Schaffeld als Justiziar des Bundesverbandes Deutscher Zeitungsverleger e.V. und der allseits einschlägig bekannte Hamburger Rechtsanwalt Prof. Dr. Matthias Prinz - aus deren Stellungnahmen (soweit sie dem RA schriftlich vorlagen bzw. nachgereicht wurden; das Ausschuss-Protokoll ist für 12/03 angekündigt) wird ggf. im Original zitiert.

16 Prinzipielle Zustimmung zu einem solchen Gesetzesvorhaben bereits bei Manzewski (SPD, in BT-PlenProt 14/221 S.22005 f.), nahezu euphorisch gar Pau (PDS, aaO S.22008), eher zurückhaltend aber noch die Grünen (Beck aaO S.22007).

17 Kienapfel (s.o. Anm.4) S.45: »Straflücken in immer größerer Zahl und rascher Folge wurden sichtbar«, vgl. Schünemann ZStW 1978, 33, NK-Jung vor § 201 Rn.3, grundlegend Peglau ZRP 1998, 249; diff. Henkel DJT 42 D 94. 
dafür erforderliche Begründung obsolet - vielmehr muss immer darauf abgestellt werden, ob es für den beabsichtigten strafrechtlichen »Schutz« einen legitimen Zweck gibt (Legitimität, s.u. 2.), ob die angestrebte Lösung ggf. geeignet ist, diesen Schutz zu bieten (Funktionalität, s.u. IV.), ob sie auch erforderlich ist, weil andere - insb. nichtstrafrechtliche - Mittel dazu nicht zur Verfügung stehen bzw. gestellt werden können (Subsidiarität, s.u. III. 3.), sowie schließlich ob sich die angestrebte Kriminalisierung nicht als unverhältnismäßig - insb. als Verstoß gegen das Übermaßverbot - erweist (Proportionalität, s.u. V.). ${ }^{20}$

\section{Lückenhafter Diskurs}

In den vorliegenden Begründungen zu den Gesetzentwürfen wird - auch insofern weitgehend übereinstimmend (Ausnahme: E-Bayern) - darauf verwiesen, Sinn der beabsichtigten Regelung sei es, »die für den Bürger in einer freiheitlichen Demokratie bestehende Notwendigkeit eines effektiven, lückenlosen Schutzes sicherzustellen. Die Unverletzlichkeit des persönlichen Lebens- und Geheimbereiches ist ein selbständiges, hochrangiges Rechtsgut, welches eines besonderen Schutzes bedarf. Denn nur dann kann der Einzelne sich zu einer verantwortungsvollen Persönlichkeit entwickeln, wenn ihm hierfür ein freier Raum vor der Gemeinschaft, dem Staat sowie anderen einzelnen Personen gewährleistet wird. Das Recht jeder Person, ihren persönlichen Lebensbereich gegenüber der Außenwelt zu schützen, ist in immer stärkerem Maße bedroht « (E-Union S.3, nahezu identisch mit E-FDP S.3).

Dieser verfassungsrechtspolitischen Analyse mag man aus bürgerrechtlicher und kriminalpolitischer Sicht nahezu ${ }^{21}$ uneingeschränkt zustimmen - und reibt sich sogleich verwundert die Augen, gingen doch in den vergangenen Jahren (und gehen weiterhin mit unverminderter Vehemenz) die gravierendsten Beeinträchtigungen des beschworenen »persönlichen Lebens- und Geheimbereichs« u.a. von jenen politischen Kräften

18 Gerade auch dieser BT-Abschnitt bietet nur einen fragmentarischen (Schmitz JA 1995, 31) bzw. sektoralen (Schünemann ZStW 1978, 31 f.) Schutz; vgl. auch NK-Jung vor § 201 Rn.3 m.w.N. und Kühl (s.o. Anm.15) S.4; allg. zur Fragmentarität des strafrechtlichen Rechtsgüterschutzes Naucke, Strafrecht. Eine Einführung, 10.Aufl. Neuwied 2002, 64 f., 193; vgl. auch Kienapfel (s.o. Anm.4) S.46 f.

19 Das ist im vorliegenden Kontext jedoch gerade nicht der Fall, denn der Gesetzgeber hat bei der Fassung der $\S \S 201 \mathrm{ff}$. StGB die z.T. geforderte und diskutierte Ausweitung des Strafrechtsschutzes auf unbefugte Bildaufnahmen (s.o. Anm.4) bewusst nicht in das StGB aufgenommen (und dieses auch bei späteren Änderungen des Abschnitts nicht etwa versehentlich unterlassen), wobei es zur Begründung hieß: »Die damit zusammenhängende Problematik ist so schwierig, daß es aus zeitlichen Gründen nicht möglich ist, in diesem Punkte eine befriedigende Lösung vorzuschlagen« (BT-Drs 7/550, 236, insoweit identisch mit VI/3250 S.225, vgl. Blei JA 1974, 603 - ähnlich E'62 S.327, vgl. Schwalm ZStW 74, 492, und AE-StGB-BT S.33).

20 Ausführlich zu diesen Verhältnismäßigkeitskriterien verfassungskonformer Gesetzgebung u.a. Paulduro, Die Verfassungsgemäßheit von Strafrechtsnormen, München 1992, Stächelin, Strafgesetzgebung im Verfassungsstaat, Berlin 1998, Sternberg-Lieben in: Hefendehl et al (Hg.), Die Rechtsgutstheorie, Baden-Baden 2003, 65 ff.; speziell zum Subsidiaritätsprinzip Kaufmann FS Henkel 1974, 89 ff., diff. Prittwitz in: Institut für Kriminalwissenschaften Frankfurt/M. (Hg.), Vom unmöglichen Zustand des Strafrechts, 1995, 387 ff. 
aus, die nunmehr die Verabschiedung der vorliegenden Gesetzentwürfe fordern: $\mathrm{Zu}$ nennen wären dabei (ohne Anspruch auf Vollständigkeit) die Ermöglichung sog. »Lauschangriffe $\ll^{22}$, die Ausweitung der Telefonüberwachung ${ }^{23}$, die Verschärfung der DNA-Analyse ${ }^{24}$ oder die Einführung öffentlicher Videoüberwachung ${ }^{25} \ldots$

Dabei soll nicht verschwiegen werden, dass - etwa im letztgenannten Bereich der Videoüberwachung - jedenfalls quantitativ die größeren Gefahren von privat oder semi-privat (resp. halbstaatlich) betriebenen Überwachungsanlagen ausgehen. ${ }^{26}$ Das ändert jedoch nichts daran, dass die jeweils betroffenen Bürgerinnen und Bürger mehr und mehr zur Kenntnis nehmen müssen, dass immer weitergehende Eingriffe in ihren »Lebens- und Geheimbereich« legalisiert werden: Ein neuer Tatbestand der »Verletzung des persönlichen Lebensbereichs « (E-Union) verspräche nicht nur viel mehr als er halten kann (und soll), er symbolisierte geradezu die vorprogrammierte bürgerrechtliche Enttäuschung, dass die subjektiv wahrgenommenen zunehmenden Verletzungen des persönlichen Lebens- und Geheimbereichs im Zweifel längst »befugt « worden sind. ${ }^{27}$ Übrig bliebe der fade Beigeschmack eines kriminalpolitischen Ablenkungsmanövers ...

21 Abgesehen von dem bereits kritisierten Anspruch einer »Lückenlosigkeit« des strafrechtlichen Schutzes ist darauf hinzuweisen, dass der persönliche Lebens- und Geheimbereich keineswegs als »unverletzlich« gilt - einen solchen absoluten Schutz genießt allenfalls die Intimsphäre (s.u. IV.2. a) und g); grundlegend zu diesen verfassungsrechtlichen Grundlagen u.a. BVerfGE 27, 1 (6 f., Mikrozensus) und 65, 1 (43 ff., Volkszählung, krit. dazu Geis JZ 1991, 113 f.).

22 Ebenso Beck BT-PlenProt 14/221 S.22007; exemplarisch dazu Leutheusser-Schnarrenberger ZRP 1998, 87 sowie Roggan (s.o. Anm.2) S.35 ff., Pinkenburg, Polizeiliche Informationsbeschaffung und Privatsphäre, Frankfurt/M. et al 2000, Zimmermann, Staatliches Abhören, Frankfurt/M. et al 2001, Mozek, Der »große Lauschangriff«, Aachen 2001 und die beeindruckende Dokumentation zu dessen Genese von Zwiehoff (Hg.), »Großer Lauschangriff «, Baden-Baden 2000.

23 Vgl. dazu Gusy ZRP 2003, 275; Überblick bei Roggan (s.o. Anm.2) S.57 ff. und KritV $2003,76 \mathrm{ff}$.

24 Exempl. dazu Braum KritV 2003, 134 ff.

25 Überblick bei Roggan (s.o. Anm.2) S.105 ff. und v. Zezschwitz in: Roßnagel (Hg.), Handbuch Datenschutzrecht, München 2003, 1876 ff.; vgl. auch Pollähne FIFF-Kommunikation 1/2002, 7 f. sowie bereits Jendro, Eingriffsqualität und rechtliche Regelung polizeilicher Videoaufnahmen, Diss. Berlin 1991 und aus der reichhaltigen aktuellen Literatur u.a.Bäumler RDV 2001, 67 ff., Roos Krim 2002, 464 ff., Schmitt-Glaeser BayVB1 2002, 584 ff., Schwarz ZG 2001, 246 ff., Pohl KJ 2003, 317 ff. und Götz FS Schreiber 2003, 103 ff.

26 So z.B. Müller MschrKrim 2002, 33; beim Hinweis auf diesen »quantitativen Sprung « wird allerdings nicht selten übersehen, dass es zur staatlichen Überwachung ein »qualitativer Sprung « ist; zur den Grenzen der Video-Überwachung am Arbeitsplatz vgl. u.a. LAG Hamm DuD 2002, 108 und HessLAG RDV 2002, 86 (dazu Tammen RDV 2000, 15), im Nachbarschafts- und Mietrecht BGH NJW 1995, 1955, LG Darmstadt NZM 2000, 360 und KG RDV 2002, 237 (dazu Horst NZM 2000, 937 ff.).

$27 \mathrm{Zu}$ den verfassungsrechtlichen Grenzen symbolischer Gesetzgebung jüngst Führ KritV 2003, 5 ff. m.w.N. 


\section{Lückenhafte Verfolgung}

In den Kommentierungen zu den $\S 201 \mathrm{ff}$. StGB - insbesondere zu $\S 203$ - ist allenthalben zu lesen, es handele sich möglicherweise um diejenigen Delikte, gegen die am häufigsten verstoßen werde, ohne dass dies irgendwelche Konsequenzen habe ${ }^{28}$. Trotz der zum Teil recht eindeutig formulierten Straftatbestände ${ }^{29}$ ist das diesbezügliche (Un)Rechtsbewusstsein offenbar völlig unterentwickelt. ${ }^{30}$ In der Praxis wird nach der Devise verfahren, die »Verstöße« (soweit sie überhaupt als solche wahrgenommen werden) seien im Zweifel legal ${ }^{31} \ldots$ und legitim ohnehin.

In der Ermittlungsarbeit der Kriminalpolizei ist dieser Deliktsbereich offenbar von so »großer « Bedeutung, dass er in der hauseigenen Kriminalstatistik überhaupt keine Erwähnung findet. ${ }^{32}$ Lediglich im Rahmen der gesteigerten Aufmerksamkeit für sog. »Computerkriminalität« werden Zahlen für das »Ausspähen von Daten « gemäß $\S 202$ a StGB genannt ${ }^{33}$, die - auf niedrigstem Niveau - erheblich schwanken, z.B. von 210 Fällen in 1999 über 538 in 2000 und 1.463 in 2001 auf 806 in 2002 ${ }^{34}$; die Zahl der ermittelten Tatverdächtigen pendelte dabei zwischen min. 266 (2000) und max. 574 (2001). Hält man (trotz der tendenziellen Unvergleichbarkeit beider Zahlenwerke) die Strafverfolgungsstatistik der Jahre 2000 und 2001 daneben, wird noch deutlicher, dass die Delikte des 15.Abschnitts in der Realität eine völlig unbedeutende Rolle spielen: So gab es - um beim PKS-Beispiel des »Ausspähens von Daten« zu bleiben - im Jahre 2000 ganze 13 Verurteilungen nach $\S 202$ a StGB und im Jahr 2001 sogar nur $5 .{ }^{35}$ Für die anderen Delikte sieht es nicht viel besser aus (vgl. Tabelle).

Soweit es also überhaupt Ermittlungsverfahren gibt, kommt es jedenfalls zu so gut wie keinen Verurteilungen (wobei der Schwerpunkt noch bei § $206 \mathrm{StGB}$ liegt). Wie gesagt: Der Hintergrund dieser Zahlen ist nicht die ausgeprägte »Rechtstreue der Bevölkerung«, sondern der nahezu völlige Ausfall der Strafverfolgung ... mit dem sich

28 Von strafrechtlichen Konsequenzen ganz zu schweigen, vgl. etwa NK-Jung vor $\S 201$ Rn.7; im Hinblick auf diesen Deliktsbereich gewinnen die Überlegungen von Popitz »Über die Präventivwirkung des Nichtwissens « (Tübingen 1968) eine besondere Brisanz, dienen die Tatbestände doch vorgeblich der Absicherung des Nichtwissens (i.S.e. Verhinderung des Zuvielwissens), während das Wissen um die hohen Dunkelziffern weit verbreitet ist.

29 Schünemann ZStW 1978, 13.

30 Peglau ZRP 1998, 250.

31 Überblick zu den Rechtfertigungsmöglichkeiten bei Wölfl Jura 2000, 231 ff.

32 Peglau ZRP 1998, 249.

33 Dazu auch Ernst NJW 2003, 3235 ff. und Schmitz JA 1995, 478 ff. sowie Hilgendorf JuS 1996, $511 \mathrm{ff}$. und $702 \mathrm{ff}$.

34 Ein im Rahmen der gesamten sog. »Computerkriminalität« im Übrigen völlig nebensächliches Deliktsfeld mit einem Anteil zwischen 0,9 und $1,8 \%$.

35 In der Diskrepanz wird deutlich, dass es offenbar überhaupt nicht um Kriminalisierung geht, eher schon um die Instrumentalisierung strafprozessualer Zwangsmittel (insb. Beschlagnahme); so dürfte auch der Hinweis von Hügel (s.o. Anm.15 S.4) zu verstehen sein, der zivilrechtliche Schutz komme als Alternative zu einer Strafbarkeit nach § 201a StGB nicht in Betracht, weil es »für den Geschädigten schwierig, wenn nicht gar unmöglich [ist], den Täter namhaft zu machen, abgesehen von dem Beweis- und Kostenrisiko« ähnlich offenbar Kühl (aaO S.6) mit dem Hinweis, die staatliche Verfolgung sei »wirksamer«. 
jene Bevölkerung aber ganz offensichtlich »eingerichtet« hat. Warum dieser deprimierenden Bilanz ${ }^{36}$ noch ein weiterer $\gg z a h n l o s e r «$ Tatbestand hinzugefügt werden muss, für den es überhaupt keinen Anlass gibt anzunehmen, er würde Ermittlungsverfahren oder gar Verurteilungen in nennenswerteren Größenordnungen nach sich ziehen ${ }^{37}$, bleibt unerfindlich. In der Gesamtbilanz ist die Kriminalisierung dieses Deliktsbereichs vielmehr derart selektiv und willkürlich, dass sich die Frage nach deren (und damit auch dessen) Legitimität aufdrängt.

Tabelle: Verurteilungen nach $\S \S 201-206$ StGB (2000/2001)

\begin{tabular}{|l|c|c|}
\hline & $\mathbf{2 0 0 0}$ & $\mathbf{2 0 0 1}$ \\
\hline$\S 201$ (Verletzung der Vertraulichkeit des Wortes) & 30 & 21 \\
\hline$\S 202$ (Verletzung des Briefgeheimnisses) & 15 & 16 \\
\hline$\S 202$ a (Ausspähen von Daten) & 13 & 5 \\
\hline$\S 203$ (Verletzung von Privatgeheimnissen) & 10 & 16 \\
\hline$\S 204$ (Verwertung fremder Geheimnisse) & 6 & 1 \\
\hline$\S 206$ (Verletzung des Post-/Fernmeldegeheimnisses) & 139 & 191 \\
\hline ges. & $\mathbf{2 1 3}$ & $\mathbf{2 4 9}$ \\
\hline
\end{tabular}

Quelle: Stat. Bundesamt, Fachserie 10, R 3 (Strafverfolgung) 2000, 22 f. und 2001, 26 ff.

Über die Gründe für die Lückenhaftigkeit der Strafverfolgung lässt sich mangels empirisch abgesicherter Erkenntnisse nur spekulieren ${ }^{38}$, letztlich dürfte sie aber darauf zurückzuführen sein, dass Taten entweder gar nicht erst zur Anzeige gebracht oder aber ggf. aufgenommene Verfahren vorzeitig wieder beendet werden. Das hat auch mit der verfahrensrechtlichen Konzeption dieses Deliktsbereichs zu tun, die sich freilich für den geplanten $\S 201$ a StGB in nahezu (s.u. IV.2.h) gleicher Weise auswirken würde $^{39}$ : Die weitaus meisten Verstöße werden ohnehin von den »Opfern« überhaupt nicht wahrgenommen (absolutes Dunkelfeld); es gibt erhebliche Beweisprobleme, u.a.

36 Diese Bilanz als »deprimierend « zu charakterisieren, bedarf der Erläuterung: Deprimierend ist sicher nicht die Tatsache an sich, dass das Strafrecht nicht zur Anwendung kommt, sondern der Umstand, dass es in Anbetracht der zahlreichen Verstöße offenkundig hoch selektiv angewandt und zum Teil willkürlich instrumentalisiert wird; vgl. auch die von Kühl (s.o. Anm.15 S.2) für die Schweiz mitgeteilten Zahlen: 37 Verurteilungen in 1968-1998, davon 29 in 1991-1998.

37 Davon gehen offenbar auch die Verfasser des E-FDP und E-Union aus, heißt es doch jeweils unter $» D$. Kosten: keine«; lediglich der E-Bayern hält die »nicht näher abschätzbaren Mehrbelastungen bei den Strafverfolgungsbehörden ... im Interesse eines verbesserten Rechtsgüterschutzes « für hinnehmbar, während Helgerth (s.o. Anm.15 S.2/4) befürchtet, der E-FDP könne einen »nicht zu bewältigenden Mehraufwand für die Strafjustiz« bereiten.

38 Vgl. auch Ernst NJW 2003, 3237 zu § 202a.

39 Anders Peglau ZRP 1998, 250, der die Nichtanwendung der persönlichkeitsschützenden Tatbestände auf deren seines Erachtens materiellrechtlich »zu enge Fassung « zurückführt. 
auch wegen zahlreicher Irrtums- und Rechtfertigungsmöglichkeiten ${ }^{40}$; es handelt sich um absolute Antragsdelikte ( $\$ 205$ StGB, Ausnahme: $\$ 206$ StGB) und z.T. um Privatklagedelikte ( $\$ 374$ Abs.1 Nr.3 StPO für $§ 202 \mathrm{StGB}^{41}$ ); soweit das »öffentliche Interesse " von Bedeutung ist ( $\$ 153$ Abs.1, 153a Abs.1, $376 \mathrm{StPO})$, dürfte dies in aller Regel verneint werden; zielen die Verfahren nicht ohnehin von vorneherein darauf ab, schriftlich erledigt zu werden (Einstellung, Strafbefehl), fürchten viele »Opfer « die Öffentlichkeit einer Gerichtsverhandlung ( $\$ 169$ GVG), die dem Schutz des Lebens- und Geheimbereichs oft diametral entgegen stehen dürfte. ${ }^{42}$

\section{Rechtliche Rahmenbedingungen: Konfliktlinien und Konkurrenzverhältnisse}

Bevor auf Details des geplanten $\S 201 \mathrm{a}$ StGB in den unterschiedlichen Entwürfen eingegangen wird (s.u. IV.), gilt es die rechtlichen Rahmenbedingungen einer solchen strafrechtlichen »Lösung « ${ }^{43}$ zu skizzieren, und zwar einerseits in verfassungs- und (weil darauf explizit Bezug genommen wurde) europarechtlicher Perspektive, andererseits im Lichte des »einfachen«, das Strafrecht umgebenden Rechts sowie strafrechtsimmanenter Alternativen.

\section{Verfassungsrecht}

Die vorliegenden Gesetzentwürfe erwecken den Eindruck, als sei der beabsichtigte strafrechtliche Schutz der Intim- resp. Privatsphäre verfassungsrechtlich geboten. ${ }^{44}$ Dem kann in dieser Allgemeinheit nicht zugestimmt werden: Ein entsprechendes Kriminalisierungsgebot ergibt sich weder aus dem Grundgesetz noch aus der bemühten Europäischen Menschenrechtskonvention, denn weder aus Art.1 Abs.1 i.V.m. 2 Abs.1

40 Wölfl Jura 2000, 234, Klug FS Oehler S.402, vgl. NK-Jung vor § 201 Rn.7, ähnlich Eberle (s.o. Anm.15 S.2), der mit einer »erheblichen Anzahl von unbegründeten Strafanzeigen« rechnet.

41 Für Schöch (s.o. Anm.15 S.2) der wesentliche Grund dafür, dass z.B. § 33 KUG praktisch irrelevant ist, ähnlich Paschke, Medienrecht, 2.Aufl. Berlin et al 2991 Rn.1025 u.a. mit Hinweis auf die vom OLG München NJW-RR 1996, 93 mitgeteilte Einstellungsentscheidung; diff. Gounalakis AfP 1998, 23, der anwaltliche Gebühreninteressen als Hintergrund vermutet.

42 Dass dieser Schutz ggf. auch über § 171b GVG hergestellt werden kann, der gerade dem Schutz der Persönlichkeitsrechte dient (Kleinknecht/Meyer-Goßner GVG § 171b Rn.1), ist dabei entweder nicht bekannt oder erscheint ungewiss, vgl. auch NK-Zaczyk $§ 185$ Rn.4 a.E.; auch Art.6 Abs.1 S.2 Hs.2 EMRK lässt einen Ausschluss der Öffentlichkeit explizit zum »Schutz des Privatlebens der Prozessparteien« zu (ähnlich Art.14 Abs.1 S.2 IPBR BGB1.II 1973, 1540).

43 Unter »B. Lösung « verzeichnen alle vorliegenden Entwürfe - in sich konsistent - die Schaffung eines neuen Straftatbestandes; unter »C. Alternativen « heißt es hingegen entweder lapidar »keine« (E-Union S.1), oder aber es wird lediglich darauf verwiesen, die Bundesregierung habe noch keinen eigenen Entwurf vorgelegt (E-FDP S.1). Den Verfassern von Gesetzentwürfen zur Ausweitung des Strafrechts sollte es - unter Verweis auf das Subsidiaritätsprinzip - zur parlamentarischen Obliegenheit gemacht werden, explizit zu begründen, warum außerstrafrechtliche Alternativen nicht in Betracht kommen (sollen); insoweit gilt für alle hier vorgelegten Entwürfe »Fehlanzeige«. 
GG noch aus Art.8 Abs.1 EMRK kann die staatliche Verpflichtung abgeleitet werden, den persönlichen Lebens- und Geheimbereich mit Mitteln des Strafrechts gegen Beeinträchtigungen durch Dritte zu schützen (gilt auch für Art.17 IPBR). Noch nicht einmal hinsichtlich des verfassungsrechtlich vorrangigen Schutzes gegen staatliche Eingriffe folgt aus den Grund- und Menschenrechten ein Gebot strafrechtlicher Abwehrmaßnahmen. Die andauernden und m.E. zunehmenden Versuche, unter Verweis auf die Grundrechte - und insbesondere ein vermeintliches »Supergrundrecht auf Sicherheit ${ }^{45}$ - die Ausweitung des Strafrechts als Ausfluss sog. »Schutzpflichten « zu propagieren $^{46}$, sind kriminalpolitisch und nicht zuletzt bürgerrechtlich zurückzuweisen (selbst wenn sie wie im vorliegenden Kontext vorgeblich darauf abzielen, anderweitigen Beeinträchtigungen von Bürgerrechten zu begegnen ${ }^{47}$ ).

Erweist sich die beabsichtigte Regelung mithin aus verfassungsrechtlichen Gründen keinesfalls als geboten oder gar unverzichtbar, so wäre ihre Einführung auf der anderen Seite allerdings ebensowenig verfassungswidrig ${ }^{48}$ - solange die o.g. Kautelen (Legitimität, Funktionalität, Subsidiarität, Proportionalität) hinreichend Beachtung fänden, die Regelung also insbesondere geeignet und erforderlich wäre (was allerdings in keinem der vorliegenden Entwürfe durchgängig gewährleistet ist, s.u. IV.). Um dabei nicht missverstanden zu werden: Der verfolgte Zweck - also der (bessere) Schutz ${ }^{49}$ der Bürgerinnen und Bürger vor unbefugten Eingriffen in ihren persönlichen Lebens- und Geheimbereich - ist zweifellos ein legitimer. ${ }^{50}$ Allerdings müssen auch

44 Ebenso Peglau ZRP 1998, 250 sowie ders. (s.o. Anm.4) S.47 ff., allerdings ist dessen Hinweis auf Art.1 Abs.1 GG einerseits und BVerfGE 39, 1 (46 f. zu $§ 218$ StGB a.F.) andererseits für sich genommen noch nicht geeignet, auch für den Bildnisschutz eine Pönalisierungspflicht zu begründen (vgl. auch abw. Voten in BVerfGE 39, $68 \mathrm{f}$. und $73 \mathrm{ff}$.), ungenau insoweit NK-Jung vor § 201 Rn.3 sowie Kühl (s.o. Anm.15) S.4: »geboten, jedenfalls aber legitimiert«; zur Konzeption des allgemeinen Persönlichkeitsrechts im GG exempl. Jarass NJW 1989, 857 ff.

45 Exemplarisch Isensee, Das Grundrecht auf Sicherheit, 1983 und Robbers, Sicherheit als Menschenrecht, 1987; zu den grundrechtlichen Schutzpflichten des Staates Klein NJW 1989, 1633 ff. (insb. S.1637 f. zu den Grenzen strafrechtlicher Schutzpflichten), diff. Dreier Jura 1994, 512 f., AK-GG-Denninger vor Art.1 Rn. 33 f., Lisken ZRP 1994, 49 ff., Gusy DÖV 1996, 573 ff., Calliess ZRP 2002, 1 ff., Albrecht KritV 2003, 128 f.; vgl. auch Leutheusser-Schnarrenberger ZRP 1998, 91 zum Lauschangriff.

46 Allgemein zu den Schutzpflichten im Zusammenhang mit dem »Persönlichkeitsschutz in der Informationsgesellschaft« Wanckel 1999, 255 ff., vgl. auch Klug FS Sarstedt 1981, 104; zu etwaigen Schutzpflichten aus Art.1 Abs.1 S.2 GG v. Münch/Kunig, GG-Kommentar Bd.1, 5.Aufl. München 2000 Art.1 Rn.31 f. sowie grundlegend Schilling KritV 1999, $452 \mathrm{ff}$. und Streuer, Die positiven Verpflichtungen des Staates, Baden-Baden 2003. Auch BT-Drs 7/550 S.235 nahm zur Begründung der Einführung des 15.Abschnitts in das StGB auf Art.1 GG und Art.8 EMRK Bezug, erhob jedoch nicht den Anspruch eines lückenlosen Schutzes; der »durch Art.1 GG auferlegten Pflicht« kann der Gesetzgeber auch zivilrechtlich nachkommen (vgl. BT-Drs 3/1237, 7 f. zur »Neuordnung des zivilrechtlichen Persönlichkeits- und Ehrenschutzes « mit einem GesE, der allerdings nie verabschiedet wurde, vgl. Schwalm ZStW 74, 490/492, ähnlich bereits Nipperdey und Larenz in DJT 42 D 3 ff. sowie die dort gefassten Beschlüsse auf D 155).

47 Ähnlich Jendro (s.o. Anm.25) S. 202 zur Videoüberwachung.

48 Vgl. zu dieser Differenzierung auch Kühl (s.o. Anm.15) S.4 ff. unter Verweis auf die Kategorien der Strafwürdigkeit und -bedürftigkeit. 
die nicht minder legitimen Zwecke Beachtung finden, die ggf. mit den »Eingriffen « verfolgt werden, also insbesondere die Presse- (Art.5 Abs.1 GG) und Kunstfreiheit (Art.5 Abs.3 S.1 GG) sowie die Berufsfreiheit (Art.12 Abs.1 S.1 GG). ${ }^{51}$ Ob dieses latente Spannungsverhältnis erst auf der Rechtswidrigkeitsebene zu lösen ist, wird noch zu klären sein (s.u. IV. 2.h).

\section{Europarecht}

Die explizite Bezugnahme auf das europäische Recht kann nicht unkommentiert bleiben, und sei es »nur« um der Gefahr vorzubeugen, vermeintlich bindende europarechtliche Vorgaben als kriminalpolitisches »Totschlagsargument« einzusetzen. Die in nahezu allen vorliegenden Entwürfen (Ausnahme: E-Bayern) erwähnte EU-Richtlinie 2002/58 vom 12.7.2002 $2^{52}$ ist bei näherem Hinsehen gar nicht geeignet, die Notwendigkeit der vorgelegten Entwürfe resp. der vorgeschlagenen strafrechtlichen Ergänzung zu belegen. Angefangen damit, dass sich diese Richtlinie auf den Datenund Privatsphärenschutz »in der elektronischen Kommunikation « beschränkt ${ }^{53}$, berührt sie den vorliegenden Problemkomplex unbefugter Bildaufnahmen überhaupt nicht, geht es doch ausschließlich um den Schutz der Kommunikationsteilnehmer und -nutzer; die in Bezug genommene Passage lautet:

»Für öffentliche Kommunikationsnetze sollten besondere rechtliche, ordnungspolitische und technische Vorschriften zum Schutz der Grundrechte und Grundfreiheiten natürlicher Personen und der berechtigten Interessen juristischer Personen erlassen

$49 \mathrm{Zu}$ den Grenzen des Selbstschutzes u.a. Kühl (s.o. Anm.15 S.5 f. m.w.N.) sowie grundlegend die viktimologische Konzeption zu $\S \S 201 \mathrm{ff}$. StGB bei Schünemann ZStW 1978, 32 ff.

50 Vgl. o. II.2.; über die Legitimität eines strafrechtlichen Beitrages (!) zu diesem Schutz mag »wohl immer« ein Konsens bestanden haben (so jedenfalls Rogall FS Hirsch 1999, 668 m.w.N.) - über dessen »Verstärkung« (aaO) aber sicher nicht, vgl. Peglau (s.o. Anm.4) S.43 ff., a.A. Arzt (s.o. Anm.4) S.340. Im Übrigen schwindet die Legitimität eines solchen Schutzes in dem Maße, wie die Betroffenen im Einzelfall selbst ihren »Lebens- und Geheimbereich « medienwirksam inszenieren (vgl. auch Fahrenhorst ZEuP 1998, 89) und zur »Proliferation von Privatem « beitragen (Rogall aaO S.690); exempl. zum »Eheleben des Kanzlers « LG Berlin AfP 2003, 174 mit berechtigter Kritik von Eggert aaO S.176 f., vgl. auch die Beiträge in Hahn (Hg.), Öffentlichkeit und Offenbarung. Eine interdisziplinäre Mediendiskussion, Konstanz 2002, darin insbesondere Neumann-Braun, InternetKameras/Web-Cams - die digitale Veröffentlichung des Privaten (aaO S.175 ff.).

51 Vgl. auch Palandt-Thomas § 823 Rn.189 ff. sowie Richter NJW 1990, 2097, Prinz ZRP 2000, 139 f., Walter ZUM 2002, 888, Soehring/Seelmann-Eggebrecht NJW 2000, 2472, Klug FS Oehler S.397 ff., Wenzel, Das Recht der Wort- und Bildberichterstattung, 4.Aufl.Köln 1994, 11 ff. und Delp, Das Recht des geistigen Schaffens in der Informationsgesellschaft, München 2003, 165 (»Antagonismus«) sowie Fahrenhorst ZEuP 1998, 87 ff. zu Art.10 EMRK im »Paparazzi«-Kontext; massive Bedenken gegen die geplanten Gesetze deshalb auch bei Eberle (s.o.Anm.15 S.2). Zur andauernden Auseinandersetzung über die Zulässigkeit von Fernsehaufnahmen (resp. deren Umfang) in Gerichtsverhandlungen im Lichte des Art.5 GG vgl. nur BVerfG NJW 2000, 2890, NJW 2001, 1633, NJW 2002, 2021 und NJW 2003, 2523 und 2671.

52 EU-ABl. L 201 v. 31.7.2002 S.37 - übrigens durchweg fälschlich als RL 2002/56 bezeichnet, ein weiterer Beleg dafür, dass das Original gar nicht gelesen (weil nicht gefunden) wurde, stattdessen hat man falsche Sekundärzitate kolportiert. 
werden, insbesondere im Hinblick auf die zunehmenden Fähigkeiten zur automatischen Speicherung und Verarbeitung personenbezogener Daten über Teilnehmer und Nutzer. $\ll^{54}$

Die Aufforderung ist also weder einschlägig ${ }^{55}$ noch macht sie überhaupt Vorgaben für die zur Umsetzung einzusetzenden Steuerungsmittel: Zunächst rangieren rechtliche gleichrangig neben ordnungspolitischen und technischen Vorschriften, und ob innerhalb der Bandbreite ggf. für erforderlich gehaltener rechtlicher Vorschriften auf das Strafrecht zurückgegriffen wird oder nicht, bleibt vollends den Mitgliedsstaaten überlassen. ${ }^{56}$ Auch wenn in einigen Politikbereichen (gerade auch der sog. »inneren Sicherheit«) immer häufiger versucht wird, der Strafrechtssetzung in den Mitgliedsstaaten europarechtliche Vorgaben zu machen, was zumindest in den Strafrechtswissenschaften mit wachsender Skepsis ${ }^{57}$ beobachtet wird: Für die Frage der Einführung einer Strafrechtsvorschrift zum Schutz vor unbefugten Bildaufnahmen ist die EU-RL 2002/58 ohne Bedeutung.

\section{Konkurrierendes Recht}

Wenn weder Verfassungs- noch Europarecht die argumentativen Lücken wettmachen: Bliebe am Ende doch nur der Verweis auf die »Lücke« im 15.Abschnitt des StGB-BT? Wie kann den im Einzelfall betroffenen Bürgerinnen und Bürgern erklärt werden, warum zwar jedem die unbefugte Verletzung der Vertraulichkeit ihres Wortes $\left(\S 201^{58}\right)$ und ihrer Briefe $\left(\S 202^{59}\right)$ sowie das Ausspähen ihrer Daten $\left(\S 202 \mathrm{a}^{60}\right)$ unter

53 Ebenso bereits die Datenschutz-RL 97/66/EG von 1997 (ABl. L 24 v. 30.11.1995); vgl. zur Deregulierung und Datensicherheit in Europa auch die Beiträge in Büllesbach (Hg.), Datenschutz im Telekommunikationsrecht, Köln 1997 sowie Wissmann (Hg.), Telekommunikationsrecht, Heidelberg 2003, 745 ff. (insb. S.757 ff.) und Eberle/Rudolf/Wasserburg (Hg.), Mainzer Rechtshandbuch der Neuen Medien, Heidelberg 2003, 329 ff. (insb. 358 f.) sowie zum strafrechtlichen Cyber-Datenschutz in Europa Kugelmann TMR 2002, 16 f. und Scheffler in: Gounalakis (Hg.), Rechtshandbuch Electronic Business, München 2003, § 35 Rn.36 ff.

54 EU-ABl. L 201 vom 31.7.2002, Ziff.7.

55 Zwar erscheint Art.1 Abs.1 der RL zunächst offen, wenn von der Zielsetzung die Rede ist, einen »gleichwertigen Schutz ... insb. des Rechts der Privatsphäre, in Bezug auf die Verarbeitung personenbezogener Daten im Bereich der elektronischen Kommunikation ... zu gewährleisten«; aus den Detailregelungen (insb. Art.5 ff.) sowie den vorangestellten Erwägungen (s.o.) geht jedoch eindeutig die Reduzierung auf den Schutz der »Nutzer « hervor (vgl. Def. in Art.2a) - für das Teledienstedatenschutzgesetz (TDDSG) vom 22.7.1999 (zul. geänd. am 21.12.2001, BGBl.I, 3721) gilt nichts Anderes (vgl. § 1 Abs.1 S.1); allg. zu diesem Problemkreis Wanckel (s.o. Anm.46) sowie Gounalakis/Rohde, Persönlichkeitsschutz im Internet, München 2002, $118 \mathrm{ff}$.

56 Vgl. auch Wanckel (s.o. Anm.46) S.281; im Übrigen kann aus dem Aufforderungscharakter einer solchen Richtlinie keinesfalls geschlossen werden, deren Verfasser hielten die deutsche Rechtslage insoweit für unzureichend; vgl. auch Gerlach AfP 2001, 1 ff. (7) zu den persönlichkeitsrechtlichen Standards in Europa.

57 Exempl. die von Albrecht et al KritV 2001, 279 ff. angestoßene Diskussion sowie die in KritV Heft 2/1999 gesammelten Beiträge zum »Corpus Iuris«, vgl. auch Albrecht KritV 2003, $125 \mathrm{ff}$. 
Strafe verboten ist, ebenso wie den jeweils genannten Personengruppen auch die Verletzung ihrer Privat- $\left(\S 203^{61}\right)$ und Post- oder Fernmeldegeheimnisse $\left(\S 206^{62}\right)$ - nicht jedoch die Verletzung ihres persönlichen Lebens- und Geheimbereichs durch unbefugte Bildaufnahmen? Liegt nicht der Umkehrschluss nahe, in Anbetracht der offenkundigen Lücke ${ }^{63}$ sei gerade diese Form der Beeinträchtigung von Persönlichkeitsrechten implizit erlaubt? Solche allzu geläufigen Suggestivfragen lenken den Blick allzu zwangsläufig auf das (Kern)Strafrecht ... als wäre alles »erlaubt«, was nicht strafrechtlich sanktioniert ist. ${ }^{64}$ Auch wenn damit die vermeintliche StGB»Lücke« - deren Umfang im Übrigen erst noch zu bestimmen wäre ${ }^{65}$ - nicht hinrei-

58 Ausf. dazu Wormer, Der strafrechtliche Schutz der Privatsphäre vor Mißbräuchen mit Tonaufnahme- und Abhörgeräten, Diss. Mannheim 1977, Kattanek, Die Verletzung des Rechtes am gesprochenen Wort ..., Münster 2000 sowie Klug FS Sarstedt 1981, 101 ff.; wird eine Videoaufnahme incl. Ton hergestellt, erfasst $§ 201$ StGB dies bereits nach geltendem Recht, da insoweit Bild- und Tonträger (vgl. § 11 Abs.3 StGB) untrennbar miteinander verbunden sind.

59 Werden (befugt oder unbefugt hergestellte) Abbildungen wie Schriftstücke verschlossen oder durch eine verschlossene Behältnis gegen Kenntnisnahme geschützt, ist ihre unbefugte Öffnung oder Kenntnisnahme gemäß § 202 Abs.3 StGB wie eine »Verletzung des Briefgeheimnisses « strafbar (Blei JA 1974, 605/606); zu den Grenzen dieses Tatbestandes im Hinblick auf Speichermedien vgl. Tröndle/Fischer § 202 Rn.4 (unter Verweis auf § 11 Abs.3 StGB); zur Möglichkeit von Schmerzensgeldansprüchen im Zusammenhang mit $\S 202 \mathrm{a}$ StGB vgl. AG Frankfurt/M. RDV 2002, 86.

60 Liegt ein Bildnis bereits als Datensatz im Sinne des § 202a Abs.2 StGB vor (vgl. dazu Tröndle/Fischer Rn.3 ff. m.w.N., demnach sogar Mikrofilmdateien), ist es als »Ausspähen von Daten« gemäß § 202a Abs.1 StGB strafbar, dieses sich oder einem anderen zu verschaffen; vgl. zu § 202a StGB auch Hilgendorf JuS 1996, 511 ff. und Schmitz JA 1995, $478 \mathrm{ff}$.

61 Es versteht sich von selbst, dass das Bildnis einer Person im Einzelfall auch ein durch $\S 203$ StGB geschütztes Geheimnis darstellen kann, vorausgesetzt, es ist dem Angehörigen einer der genannten potenziellen Tätergruppen in dessen jeweiliger Funktion bekannt geworden und (noch) »geheim«, weil es »nur einem einzelnen oder einem beschränkten Personenkreis bekannt ist « und der Betroffene an der Geheimhaltung »ein schutzwürdiges Interesse hat « (Tröndle/Fischer § 203 Rn.3 und 7 ff. m.w.N.).

62 Gemäß § 206 Abs.5 StGB unterliegt dem Postgeheimnis auch der Inhalt von Postsendungen (S.1) und dem Fernmeldegeheimnis auch der Inhalt der Telekommunikation (S.2), also ggf. auch Bildnisse, so dass darauf bezogene Tathandlungen (insb. Mitteilung gemäß Abs.1, Öffnung/Kenntnisverschaffung gemäß Abs.2 Nr.1) der jeweils bezeichneten Täterkreise einen weiteren Ausschnitt des bereits existierenden strafrechtlichen Bildnisschutzes darstellen.

63 So auch Kühl (s.o. Anm.15) S.4 f. und Schöch (aaO) S.2, vgl. bereits Lenckner FS Baumann 1992, 139, jeweils m.w.N.

64 Allzu leicht wird in solchen und vergleichbaren Diskussionen - wissentlich oder (was mindestens so schwer wiegt) aus Unkenntnis - ignoriert, dass es nicht nur das Ordnungswidrigkeiten- und allgemeine Ordnungsrecht gibt, das deutlich mehr Verbote bereit hält als das Strafrecht, sondern auch zahlreiche zivilrechtliche Verhaltensregeln, die vollstreckungsrechtlich und ggf. prozessual durchgesetzt werden können (vgl. dazu am Beispiel des Gewaltschutzgesetzes Pollähne in: Barton (Hg.), Verfolgen - Vermitteln - Verklagen. Dokumentation der 2. Bielefelder Verfahrenstage, Baden-Baden 2004, in Druck). 
chend erklärt sein mag ${ }^{66}$, bedarf es doch einiger Seitenblicke auf anderweitige rechtlich einschlägige (auch nebenstrafrechtliche) Regelungen ${ }^{67}$ :

\section{a) Zivilrecht}

Im Zivilrecht wird das sog. »Recht am eigenen Bild « spezialgesetzlich geschützt: Jenseits bürgerlich-rechtlicher Beseitigungs-, Unterlassungs-, Ersatz- und ggfls. Schmerzensgeldansprüche ${ }^{68}$ wegen Verletzung des allgemeinen Persönlichkeitsrechts gemäß $\S \S 823,1004 \mathrm{BGB}^{69}$ greifen als urheberrechtliche Spezialregelungen ${ }^{70}$ zum Bildnisschutz die $\$ \S 22$ ff. des sog. »Kunst-Urhebergesetzes « (KUG). ${ }^{71}$ Danach dürfen Bildnisse »nur mit Einwilligung des Abgebildeten verbreitet oder öffentlich zur Schau gestellt werden« $(\S 22$ S.1 KUG), Zuwiderhandlungen sind gemäß $§ 33$ KUG strafbewehrt. Die ggfls. unbefugte Herstellung der Bildnisse wird durch das KUG zwar grundsätzlich nicht erfasst ${ }^{72}$, aber immerhin ein kleinerer Ausschnitt des geplanten $\S 201 \mathrm{a}$ StGB (Gebrauchen, Zugänglichmachen) ist bereits durch $\S 33$ KUG weitgehend abgedeckt. ${ }^{73}$

65 In den vorangegangenen Anmerkungen zu den $\S \S 201 \mathrm{ff}$. StGB dürfte deutlich geworden sein, dass die Lücke im strafrechtlichen Bildnisschutz nicht so groß ist, wie behauptet wird, und sich letztlich auf bestimmte Formen der Bildaufnahme reduziert (sieht man zunächst von dem weitergehenden Schutz vor bloßer Beobachtung ab, dazu s.u. IV.2.d.).

$66 \mathrm{Zu}$ warnen ist im kriminalpolitischen »Lücken«-Diskurs allerdings vor einer Beweislastumkehr: Nach dem Prinzip »in dubio pro libertate « (dazu exempl. Maihofer in: Benda et al (Hg.), Handbuch des Verfassungsrechts, 2.Aufl. 1994 § 12 Rn.124) muss nicht die »Lücke« legitimiert werden, sondern deren Füllung!

67 Überblick z.B. auch bei Gounalakis/Rohde (s.o. Anm.55) S.23 ff. und Peglau (s.o. Anm.4) S.26 ff.

68 Je stärker auf die Abschreckungsfunktion von Schmerzensgeldansprüchen gesetzt wird (vgl. dazu u.a. Gounalakis AfP 1998, 17 ff., 24 f., Ladeur ZUM 2000, 886 f. m.w.N. auch zum Thema »Kommerzialisierung der Person«, vgl. BGH NJW 1995, 861, OLG Hamburg NJW 1996, 2870, und BVerfG NJW 2000, 1021 sowie die Polemik von Seitz NJW 2000, 2167 f., ähnlich Stuhlmann, Der zivilrechtliche Persönlichkeitsschutz bei Ehrverletzung und kommerzieller Vermarktung in Deutschland, BoD 2001, $235 \mathrm{ff}$.), je deutlicher hat das Strafrecht dahinter als subsidiär zurückzutreten (Ähnliches ist im Kontext des GewSchG zu beobachten, s.u. b); allg. zu Strafe und Prävention im Bürgerlichen Recht: Schäfer AcP 2002, 397 ff.; zum Bildnisschutz in der Spruchpraxis des Deutschen Presserats vgl. auch Münch AfP 2002, 18 ff.

69 Grundlegend Helle, Besondere Persönlichkeitsrechte im Privatrecht, Tübingen 1991, Ehmann JuS 1997, 193 ff. und Geis JZ 1991, 112 ff., sowie BGHZ 24, 72, 200 (208 ff.); 26, 349 (354 ff.); 35, 363 und 39, 124 (130 ff.) m.w.N., vgl. Palandt-Thomas $§ 823$ Rn.175 ff.,195 f. und -Bassenge $\S 1004$ Rn.2 sowie Prinz/Peters, Medienrecht, München 1999 Rn.891 ff., Prinz ZRP 2000, 142 f., Walter ZUM 2002, 888 ff., Miserre JA 2003, 252 und Becker/Smeets Jura 2000, 353 ff., Fechner, Medienrecht, 4.Aufl. Tübingen 2003 Rn. 250 ff. und Gounalakis AfP 1998, 10 ff.; zu Ansprüchen analog §§ 12, 862 BGB vgl. BAG NJW 1990, 2272 sowie zum Schutz des Unternehmerpersönlichkeitsrechts KG NJW 2000, 2210 m.w.N.

70 Denen gegenüber kommen die genannten Ansprüch nach BGB nur subsidiär in Betracht, vgl. Miserre JA 2003, 254. 


\section{b) Gewaltschutzzrecht}

Ein dem Zivilrecht angelagertes neues Interventionsrecht bietet das sog. »Gewaltschutzgesetz $($ GewSchG) « mit seinem Schwerpunkt zivilprozessualer Schutzmaßnahmen (§ 1) zzgl. strafrechtlicher Absicherung (\$4) und landespolizeirechtlicher Flankierungen (Platz-/Wohnungsverweisung, Rückkehrverbot). ${ }^{74}$ Über die im Zentrum des Interesses stehenden Maßnahmen gegen sog. »häusliche Gewalt« hinaus reicht der Schutz gegen »Nachstellungen« ( 1 Abs.2 GewSchG, neudeutsch »Stalking ${ }^{75}$ ), der auch Maßnahmen gegen eine Person umfasst, die widerrechtlich und vorsätzlich »eine andere Person dadurch unzumutbar belästigt, dass sie ihr gegen den ausdrücklich erklärten Willen wiederholt nachstellt oder sie unter Verwendung von Fernkommunikationsmitteln verfolgt « (S.1 Nr.2b), und damit z.B. auch gegen sog. »Paparazzi« eingesetzt werden kann. ${ }^{76}$ Verstößt der Täter gegen solche zivilgerichtlichen Schutzanordnungen (z.B. gegen das Verbot, sich »in einem bestimmten Umkreis

71 Ausf. dazu Engels/Schulz AfP 1998, 574 ff.; der noch immer geläufige Begriff »KunstUrhebergesetz « (KUG) ist insofern irreführend, als die meisten Vorschriften dieses aus dem Jahr 1907 stammenden »Gesetzes betreffend das Urheberrecht an Werken der bildenden Künste und der Photographie « (vgl. zur Entstehung Walter ZUM 2002, 889 Fn.33,39 und Schulz/Jürgens JuS 1999, 664 sowie Henkel DJT 42 D 87 ff.) längst aufgehoben worden sind, insbesondere jene, die das Urheberrecht betreffen, das abschließend im UrhG geregelt ist - genau genommen handelt es sich bei dem Rest-KUG gar nicht mehr um Urheberschutzrecht, sondern ausschließlich um Persönlichkeitsschutzrecht gegen die Urheber von Bildnissen (vgl. auch Delp, s.o. Anm.51 S.164: Fremdkörper); bezeichnenderweise war der Persönlichkeitsschutz seinerseits auch nicht Gegenstand des jüngst in Kraft getretenen »Gesetzes zur Regelung des Urheberrechts in der Informationsgesellschaft « vom 10.9.2003 (BGBl.I, 1774, vgl. dazu Czychowski NJW 2003, 2409).

72 Mestmäcker/Schulze, Kommentar zum Urheberrecht, Bd.2 KSchG § 22 Anm.2, Wenzel (s.o. Anm.51) S.337 ff., Loewenstein, Handbuch des Urheberrechts, München $2003 \S 18$ Rn.1,2,7, Walter ZUM 2002, 889 Fn.39 und 891 Fn.52, Peglau ZRP 1980, 250, vgl. OLG Celle JR 1979, 422. Soweit $\$ 37$ KUG dadurch Verwirrung stiftet, dass von einem Anspruch auf Vernichtung hergestellter Exemplare und Werke die Rede ist, bezieht sich dies auf ehemalige Teile des Gesetzes, die nicht mehr gelten; in $\S 17$ Abs.4 BGB der BTDrs 3/1237 sollte die Bildanfertigung in die Regelungen einbezogen (vgl. aaO S.22 f.), zugleich aber der Strafrechtsschutz des § 33 KUG aufgehoben werden, »da er, wie eine Umfrage ergeben hat, geringe praktische Bedeutung hat « (aaO S.31) - dieser GesE wurde jedoch nie verabschiedet.

73 Hierauf wird z.T. unter IV. noch einmal präzise eingegangen, vgl. auch OLG Oldenburg NJW 1963, 922, Wenzel (s.o. Anm.51) S.377 und Helle (s.o. Anm.69) S.225, der bzgl. einer Strafbarkeit nach $\S 33$ iVm 23 Abs.2 KUG Bedenken im Hinblick auf Art.103 Abs.2 GG äußert; umgekehrt würde § 33 KUG durch Einführung eines § 201a StGB weitgehend gegenstandslos - für die Einstellung des § 33 KUG ins StGB Rogall FS Hirsch 1999, 692, ähnlich offenbar Schöch (s.o. Anm.15 S.2/3), krit. zur Weite des Tatbestandes allerdings bereits Henkel DJT 42 D 92 f.; allg. zum Verhältnis Zivilrecht vs. Strafrecht Rogall aaO und Arzt (s.o. Anm.4) S.311 ff.

74 Überblick bei Schweikert/Baer, Das neue Gewaltschutzrecht, Baden-Baden 2002 und Löhnig/Sachs, Zivilrechtlicher Gewaltschutz, Berlin 2002 sowie Pollähne (s.o. Anm.64).

75 Dazu Pollähne NK 2002, 56 und StV 2003, 563 m.w.N., zu Kontaktverboten zum Schutz vor »Stalking « auch Kaboth ZUM 2003, $342 \mathrm{ff}$.

76 Schwab FamRZ 2002, 3, Grziwotz NJW 2002, 873, vgl. auch Walter ZUM 2002, 886 ff. 
der Wohnung der verletzten Person aufzuhalten«, Abs.1 S.3 Nr.2) macht er sich strafbar $(\S 4) .{ }^{77}$ Ein nicht unerheblicher Anteil der im Kontext eines neuen $\S 201 \mathrm{a}$ StGB in

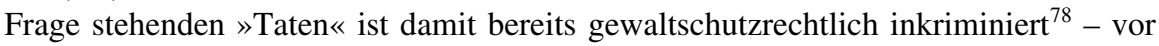
Schaffung eines weiteren Straftatbestandes sollten die Erfahrungen mit dem neuen GewSchG abgewartet und ausgewertet werden. ${ }^{79}$

\section{c) Datenschutzrecht}

Aus dem öffentlichen Datenschutzrecht sind die Rechte der Betroffenen gegen staatliche Maßnahmen gemäß $\S 19$ ff. BDSG und gegenüber anderen Stellen gemäß $\S 33$ ff. $\mathrm{BDSG}^{80}$ in die Betrachtung einzubeziehen. Die gesetzlichen Verpflichtungen sind ordnungsrechtlich ( $\$ 43$ BDSG) und strafrechtlich (§ 44 i.V.m. $§ 43$ Abs.2 BDSG) abgesichert; zudem können datenschutzrechtliche Verstöße auch Ersatzansprüche gemäß $§ 823$ Abs.2 BGB begründen. ${ }^{81}$ Damit ist sanktionsbewehrt insb. untersagt, »unbefugt personenbezogene Daten, die nicht allgemein zugänglich sind «, zu erheben oder zu verarbeiten ( 93 Abs. 2 Nr.1 BDSG, ähnlich $§ 33$ Abs.1 DSG-NW).

Jenseits der Frage, in welchen Konstellationen das jeweilige Datenschutzrecht überhaupt Anwendung findet ${ }^{82}$, kommen Maßnahmen gegen die Aufzeichnung und Verarbeitung von Bildern in Betracht, soweit es sich dabei um »Einzelangaben über

77 Ausf. zu den Problemen der Strafbarkeit nach § 4 GewSchG mein demnächst erscheinender Beitrag »Probleme des Gewaltschutzgesetzes aus strafrechtlicher Sicht« (s.o. Anm.64).

78 Dabei ist dem GewSchG insbesondere das Subsidiaritätsprinzip zu entnehmen, gegen »Nachstellungen« vorrangig zivilgerichtlichen Schutz (und als Sofortmaßnahmen ggfls. polizeirechtliche Hilfe) in Anspruch zu nehmen, während das Strafrecht allenfalls bei Zuwiderhandlungen gegen bereits ergangene zivilgerichtliche Schutzanordnungen eingreift; im Übrigen tritt das Strafrecht umso weiter zurück, je stärker bereits das Zivilrecht auf Intervention und Prävention setzt (vgl. Anm.61 zum Abschreckungscharakter des Schmerzensgeldes).

79 Ähnlich Franke (s.o. Anm.15 S.7); immer häufiger ist zu beklagen, dass der Gesetzgeber in immer kürzeren Abständen bestehende Regelungen ändert oder ergänzt, bevor deren Geeignetheit und Erforderlichkeit evaluiert worden ist; was auch immer vom neuen Gewaltschutzrecht zu halten sein mag (wobei neben dem GewSchG auch die neuen polizeirechtlichen Möglichkeiten Beachtung finden müssen, vgl. dazu u.a. Hermann NJW 2002, 3062 ff. und Naucke-Löhmer FPR 2002, 641 ff.), dessen Evaluation wurde von vorneherein eingeplant (vgl. dazu auch die Beiträge von Baer und Kavemann in: Barton, s.o. Anm. 64), wobei freilich dahingestellt bleibt, wie »ergebnisoffen « eine »wissenschaftliche Begleitung « ist, die von denjenigen organisiert wird, die ehedem für die Verabschiedung eben jenes Gesetzes gekämpft haben.

80 Für die weitaus meisten Fälle gilt allerdings das jeweils einschlägige Landesdatenschutzrecht, im Folgenden dargestellt am Beispiel des DSG-NW, wobei zu beachten ist, dass gerade hinsichtlich der nebenstrafrechtlichen Flankierung im Landesrecht die größten Unterschiede zu verzeichnen sind, vgl. Dammann in: Simitis, BDSG-Kommentar, 5.Aufl. Baden-Baden 2003 § 44 Rn.7; zum Datenschutz im Multi-Media-Recht vgl. die Beiträge von Helfrich, Fraenkel, Büttgen und Schmitz in Hoeren/Sieber, Handbuch Multi-MediaRecht, München 2003 Teil 16.

81 Palandt-Thomas § 823 Rn. 179.

82 Vgl. insb. §§ 1 Abs.2, 27 Abs.1 BDSG; § 2 Abs.2 S.2 DSG-NRW verweist wiederum - mit geringfügigen Abweichungen - auf die $\S \S 27$ ff. BDSG incl. der nebenstrafrechtlichen Absicherung. 
persönliche oder sachliche Verhältnisse einer bestimmten oder bestimmbaren natürlichen Person « handelt. ${ }^{83}$ Aufnahmen auf Bildträger stellen spätestens dann solche »Einzelangaben « dar, wenn sie einer bestimmten Person und bestimmten Orten und/ oder Zeiten zugeordnet werden (können). ${ }^{84}$ Auch der Gesetzgeber geht in § 6b BDSG offenbar davon aus, dass per »Videoüberwachung (Legaldefinition in Abs.1: »Beobachtung öffentlich zugänglicher Räume mit optisch-elektronischen Einrichtungen«) Daten erhoben werden (Abs.3 bis 5; ähnlich § 29b DSG-NW).

Strafbar ist die unbefugte Erhebung oder Verarbeitung, wenn sie »gegen Entgelt oder in der Absicht [begangen wird], sich oder einen anderen zu bereichern oder einen anderen zu schädigen « $(\S 44 \text { Abs.1 BDSG, } \S 33 \text { Abs.1 DSG-NW })^{85}$, wobei nicht nur der Betroffene, sondern auch die Datenschutzbeauftragten und Aufsichtsbehörden (gemäß $§ 38$ BDSG) antragsberechtigt sind (§ 44 Abs.2 BDSG, gemäß § 33 DSG-NW ganz ohne Antragserfordernis). Wiederum: Vor Schaffung eines weiteren Straftatbestandes sollte - normativ und empirisch - geklärt werden, ob jenseits der $\S \S 43,44$ BDSG (resp. der entsprechenden landesdatenschutzrechtlichen Regelungen) im Zusammenhang mit unbefugten Bildaufnahmen und deren Verarbeitung Fälle strafwürdigen Unrechts verbleiben. ${ }^{86}$

\section{d) (Kern)Strafrecht}

Schließlich darf nicht in Vergessenheit geraten, dass die vermeintlich strafwürdigen Verhaltensweisen zum Teil bereits im sog. Kernstrafrecht erfasst sind, wobei insbesondere die Strafbarkeit des Hausfriedensbruchs gemäß $§ 123$ StGB und der Beleidigung gemäß $\S 185$ StGB zu nennen und die Interdependenzen mit dem Sexualstrafrecht zu beachten sind ${ }^{87}$ :

Zwar liegt ein Hausfriedensbruch in der Version des Eindringens nur vor, wenn der Täter »zumindest mit einem Teil seines Körpers in den geschützten Raum gelangt ist ${ }^{88}$, so dass insbesondere das »Eindringen « in die häusliche Intim- oder Privatsphäre

$83 \S 3$ Abs.1 BDSG bzw. DSG-NW, erhebliche Bedenken dagegen unter Verweis auf Art.103 Abs.2 GG bei Schünemann ZStW 1978, 23 ff. m.w.N.; zum diff. Datenbegriff des § 202a Abs.2 StGB vgl. Tröndle/Fischer § 202a Rn.3 f. m.w.N. und Schmitz JA 1995, 478 ff.

84 Vgl. Dammann (s.o. Anm.79) § 2 Rn.4 und 111: Bildaufnahmen als Datenerhebung; ähnlich Amelung, Der Schutz der Privatheit im Zivilrecht, Tübingen 2002, 161, vgl. auch Legler CR 1998, 442.

85 Zur Auslegung des Regelungsmerkmals »schädigen« verweist Dammann (s.o. Anm.79) $\S 44 \mathrm{Rn} .8$ auf den insofern wortgleichen $\S 203$ Abs.5 StGB, wonach auch immaterielle Schädigungen erfasst seien (etwa Bloßstellungen), vgl. Tröndle/Fischer $§ 203 \mathrm{Rn} .50$ (seinerseits mit Verweis auf die Nachteilszufügungsabsicht in $\S 274$ StGB, dort Rn.6).

86 Dass der Bundesdatenschutzbeauftragte selbst gefordert hat, diesbezüglich das StGB in Anschlag zu bringen (BT-Drs 14/5555, 22), ist für sich genommen noch kein hinreichender Beleg für die Unzulänglichkeit des BDSG, zumal zu erwarten gewesen wäre, dass er sich zur etwaigen Unzulänglichkeit »seiner« §§ 43, 44 BDSG zumindest äußert.

87 Neben den bereits erwähnten Tatbestandsvarianten der $\S \S 201$ ff. StGB (s.o. vor a) kommen für Ausnahmefälle unbefugter Bildaufnahmen noch weitere Tatbestände in Betracht, wie etwa die $\S \S 167$ ff. StGB (grob störende Aufnahmen bei der Religionsausübung, bei Bestattungsfeiern oder der Totenruhe), vgl. auch den Überblick bei Peglau (s.o. Anm.4) S.26 ff. und Sieber in Hoeren/Sieber (s.o. Anm.80) Teil 19 Rn.416 ff. 
mit einer Kamera oder vergleichbaren Mitteln nicht vom Tatbestand erfasst wird, gleichwohl dürfte ein Teil der fraglichen Fälle durch $\S 123$ StGB abgedeckt sein, zumal insbesondere das Grundstück des Opfers, von dem aus ggfls. Aufnahmen hergestellt werden, bereits zu dem durch die Vorschrift geschützten »befriedeten Besitztum « gehört. $^{89}$

Eine Beleidigung kann in unbefugten Bildaufnahmen gesehen werden, wenn sie als konkludentes Handeln eine sog. »Kundgabe der Missachtung « im Sinne einer gedanklichen Äußerung aufweisen ${ }^{90}$, was freilich auf Ausnahmefälle beschränkt bleibt, denn ein Verhalten, das sich letztlich auf Informationsgewinnung reduziert ${ }^{91}$, kann schwerlich als Äußerung gewertet werden. ${ }^{92}$ Eine sog. »sexualbezogene «Beleidigung, auf die in den Begründungen explizit Bezug genommen wird, kann im vorliegenden Kontext allenfalls darin liegen, dass in dem sexualbezogenen Verhalten des Täters zugleich eine herabsetzende Bewertung des Opfers zu sehen ist ${ }^{93}$, was jedoch bei bloßem Beobachten in aller Regel ebenfalls nicht angenommen werden $\mathrm{kann}^{94}$ : Sog. »Spanner«, die es zumeist gerade nicht darauf anlegen, von der beobachteten Person bemerkt zu werden, begehen keine Beleidigung. ${ }^{95}$ Etwas Anderes kann gelten bei sexualbezogenen Aufnahmen, die auch eine entsprechende Verwendung finden sollen. ${ }^{96}$

Schließlich kann bloßes - wenn auch sexualbezogenes - Beobachten keine Strafbarkeit wegen der Erregung eines öffentlichen Ärgernisses gemäß § 183a StGB

88 Vgl. Tröndle/Fischer $§ 123$ Rn.14 m.w.N. sowie bereits RGSt 73, 385.

89 Tröndle/Fischer aaO Rn.8 f. und NK-Ostendorf § 123 Rn.23 m.w.N., vgl. auch BGH StV 1997, 400; dabei darf freilich nicht übersehen werden, dass moderne Foto-, Film- und Videotechnik gestochen »scharfe « Aufnahmen in jenem »befriedeten Besitztum « ermöglicht, ohne diesem auch nur nahekommen zu müssen (zu den Grenzen des Selbstschutzes auch Kühl, s.o. Anm.15 S.5 f.). Zur Kombination von Hausfriedensbruch und der nach Scheizer Recht strafbaren Privatsphärenverletzung durch Fotoaufnahmen im Falle Barschel vgl. SchwBG MDR 1993, 783.

90 NK-Zaczyk vor $\S 185$ Rn.18 ff. und $\S 185$ Rn.2 ff.; allg. Rogall FS Hirsch 1999, 665, vgl. auch BayObLG NJW 1980, 1969, diff. BGHSt 9, 17. Dies ist nicht zu verwechseln mit einer Beleidigung per Video, vgl. dazu BayObLG NJW 2000, 1584 mit berechtigter Kritik von Wrage NZV 2001, 68.

91 Beobachten, Belauschen, Fotografieren, Filmen etc., vgl. NK-Zaczyk vor $\S 185$ Rn.22 m.w.N., diff. OLG Oldenburg NJW 1963, 920 f. für den Fall, dass die (straflos) aufgenommenen Fotos anschließend zur Kundgabe der Missachtung des Betroffenen verbreitet werden sollen.

92 Teubner JR 1979, 424, Haller/Steffens JA 1996, 656 m.w.N.; a.A. Peglau ZRP 1998, 250, der für eine »persönlichkeitsrechtsbezogene Auslegung « insb. der sog. »Formalbeleidigung « gemäß § 185 Alt.2 StGB plädiert (ausf. ders. s.o. Anm.4 S.55 ff. und 83 ff.), damit aber tendenziell Art.103 Abs.2 GG aus dem Blick verliert (vgl. auch Pollähne StV 2003, 564 m.w.N.).

93 NK-Zaczyk aaO Rn.25 und Tröndle/Fischer § 185 Rn.11.

94 Tröndle/Fischer § 185 Rn.11, deutlich OLG Düsseldorf NJW 2001, $3562=$ JMBl 2001, 265: Keine Beleidigung durch bloßes »Anstarren«, und sei es in einer »peinlichen « Situation, ähnlich die Stellungnahme von Hügel (s.o. Anm.15 S.2).

95 Anders Schöch (s.o. Anm.15 S.4) bei »massivem Voyeurismus«, was immer das sein mag; im Übrigen ist ebenfalls umstritten, ob bzw. wann exhibitionistische Handlungen gemäß $\S 183$ StGB zugleich eine Beleidigung darstellen können, vgl. Tröndle/Fischer § 183 Rn.16 m.w.N. 
begründen ${ }^{97}$, denn sog. »Voyeurismus « ist als solcher noch kein »öffentliches « Ärgernis. ${ }^{98} \mathrm{Ob}$ bereits das »Öffentlich-Machen « fremder sexueller Handlungen - z.B. durch heimliches Herstellen von Beobachtbarkeit - ausreichen soll, ist umstritten ${ }^{99}$, entsprechende Entscheidungen sind aber nicht bekannt. Auch die strafbare Herstellung pornographischer Schriften (wozu gemäß $§ 11$ Abs.3 StGB auch Bildträger und Abbildungen gehören) gemäß $\S 184$ Abs.1 Nr.8 StGB dürfte nur in seltenen und besonders drastischen Fällen eingreifen ${ }^{100}$, was gleichermaßen gilt für die Vornahme sexueller Handlungen entweder gegen den Willen der betroffenen Person oder an Kindern, Schutzbefohlenen oder ähnlichen Personen. ${ }^{101}$

Das Sexualstrafrecht hilft in den fraglichen Fällen letztlich auch deshalb nicht weiter, weil gemäß $§ 184 \mathrm{c}$ Nr.1 StGB als sexuelle Handlungen nur solche gelten, »die im Hinblick auf das jeweils geschützte Rechtsgut von einiger Erheblichkeit sind «. Äußerlich neutrale Handlungen wie z.B. das Fotografieren werden nicht schon durch die ggfls. vorhandene sexuelle Motivation des Täters, sondern erst durch eine nach außen erkennbare Sexualbezogenheit zu einer sexuellen Handlung ${ }^{102}$, die zusätzlich auch noch »einige Erheblichkeit« aufweisen müsste. Dem Reformgesetzgeber war seinerzeit daran gelegen, (auch via $\S 184 \mathrm{c}$ StGB) das Sexualstrafrecht zu entbagatellisieren und -moralisieren, indem es aus dem Dunstkreis des »Unzüchtigen und Unsittlichen« auf den strafwürdigen Bereich der Taten »gegen die sexuelle Selbstbestimmung « (so die offizielle Überschrift des 13.Abschnitts) reduziert wurde. Trotz deutlicher und z.T. drastischer Ausweitungen des Sexualstrafrechts in den vergangenen rund 10 Jahren $^{103}$ wurde an diesem Leitbild festgehalten, weshalb Tendenzen bedenklich sind, die Strafbarkeit außerhalb dieses Abschnitts durch extensive Anwendung vermeintlicher Auffangtatbestände wieder auszudehnen. ${ }^{104}$ Auch für den Fall der

96 Vgl. auch den Fall BGH NStZ 1984, 216; im Hinblick auf den geplanten § 201a StGB ist von Bedeutung, dass nicht auf die Herstellung der Aufnahme an sich, sondern erst auf deren Verbreitung abgestellt wird (ähnlich § 33 KUG, s.o.) - ist die spätere Verbreitung beabsichtigt, könnte in der Herstellung der Aufnahme in Ausnahmefällen bereits ein strafbarer Versuch liegen (sehr weitgehend in der Formulierung eines Tatbestandes der sexuellen Beleidigung ein ehemaliger GesE der Grünen in BT-Drs 11/1040,3,7).

$97 \mathrm{Zu}$ Details Tröndle/Fischer § 183a Rn.3; vgl. zur Abgrenzung des § 183 StGB auch RGSt $73,385 \mathrm{f}$.

98 Eine Strafbarkeit könnte sich nach neuer Rechtslage allerdings auf dem Umweg über das GewSchG (s.o. d) ergeben, vgl. Pollähne (s.o. Anm.77).

99 Vgl. Tröndle/Fischer § 183a Rn.3 einerseits und Sch/Sch-Lenckner/Perron § 183a Rn.7 andererseits.

100 Details und Kritik bei Tröndle/Fischer $\$ 184$ Rn.7 und 19 f. m.w.N.

101 Vgl. § 177 Abs.1 StGB einerseits und die $\S \S 174$ Abs.1, 174a, 174b, 174c, 176 Abs.1, 179 Abs.1, 182 Abs.1,2 StGB andererseits; exempl. BGHSt 43, 368 und NStE § 176 Nr.7.

102 Tröndle/Fischer § 184c Rn.3, anders z.T. noch BGHSt 17, 280.

103 Exempl. Jäger in: Institut für Kriminalwissenschaften und Rechtsphilosophie Frankfurt/M. (Hg.), Irrwege der Strafgesetzgebung, 1999, 49 ff., Kobbé/Pollähne ZfPP-SH 1999, 237 ff.; Überblick bei Laubenthal, Sexualstraftaten, Berlin et al 2000, $8 \mathrm{ff}$.

104 Vgl. etwa die Kritik von NK-Zaczyk § 185 Rn.24 an der sog. ,Sexualbeleidigung' (s.o.); probl. insofern auch die Einführung der »kleinen sexuellen Nötigung « in § 240 Abs.4 S.2 Ziff.1 StGB, vgl. dazu Tröndle/Fischer § 240 Rn.59 f. m.w.N. 
Einführung eines neuen $§ 201$ a StGB müsste gewährleistet sein, keinen Auffangtatbestand zu schaffen, der sexualbezogene Handlungen strafrechtlich erfasst, die mit guten Gründen nicht bzw. entkriminalisiert worden waren. ${ }^{105}$

e) Zwischenergebnis: Lücken?

Insgesamt bieten die dargestellten Regelungen des geltenden Rechts zwar keinen »lückenlosen« strafrechtlichen Schutz gegen Verletzungen des Lebens- und Geheimbereichs durch die unbefugte Herstellung und Verarbeitung von Bildnissen, es drängen sich aber Zweifel auf, dass die verbleibenden »Lücken « tatsächlich strafwürdiges Unrecht repräsentieren und deshalb einen neuen Straftatbestand legitimieren (der seinerseits einen lückenlosen Schutz ebenso wenig gewährleisten könnte ${ }^{106}$ ). Auch wenn dieses Zwischenergebnis geeignet sein mag, einen Teil der bereits bestehenden Regelungen des 15.Abschnitts im BT des StGB (in puncto Strafwürdigkeit) ebenfalls in Frage zu stellen ${ }^{107}$, ist allein damit die Erforderlichkeit des geplanten $§ 201$ a StGB noch nicht hinreichend dargetan.

\section{Zu den vorgeschlagenen Regelungen}

Jenseits dieser grundsätzlichen Anmerkungen, die auch die eigenen Ambivalenzen zum Ausdruck bringen sollten - Unterstützung des Schutzes der Bürgerrechte gegen Verletzungen der Privatsphäre (Legitimität) vs. kriminal- und bürgerrechtspolitische Bedenken gegen deren Kriminalisierung (Subsidiarität) - und an deren Ende letztlich die Skepsis hinsichtlich der Sinnfälligkeit der beabsichtigten strafrechtlichen Lückenfüllung überwiegt ${ }^{108}$, soll nachfolgend gleichwohl und gleichsam »systemimmanent « Stellung genommen werden zu den konkreten Regelungsvorschlägen. Leitli-

105 Vgl. auch OLG Düsseldorf NJW 2001, 3563; dass gerade diese Gefahr naheliegt, belegen die von Hügel (s.o. Anm.15 S.2) mitgeteilten Zahlen von »hochgerechnet ca. 80 bis 100 derartige Verfahren - ausschließlich mit sexuellem Bezug oder Hintergrund « allein bei der StA Konstanz, die sie nach geltendem Recht »obwohl als Unrecht empfunden, nicht verfolgen können «.

106 Das sieht wohl auch Peglau ZRP 1998, 251: »Angesichts der zahlreichen (!) strafrechtlich nicht sanktionierten Verletzungsmöglichkeiten sollte aber wenigstens (?) hinsichtlich schwerer (!) Persönlichkeitsverletzungen ein umfassender (?) Tatbestand in das StGB aufgenommen werden «; in Anbetracht z.B. der Ausführungen von Ladeur zum »ImageSchutz« im Rahmen der »Ökonomie der Aufmerksamkeit« (NJW 2000, 1977 ff. m.w.N., vgl. auch ders. ZUM 2000, 884 ff.) wäre es wohl nur eine Frage der Zeit, bis von irgendeiner Seite (gerade auch von selbst betroffenen Politikern) eine entsprechende Ausweitung des $\S 201$ a StGB gefordert wird - eklatante Einzelfälle sind bekanntlich ebenso schnell gefunden wie kriminalpolitisch instrumentalisiert, wie dies bereits beim 25.StÄndG zu sehen war, als sich der Gesetzgeber »durch einige spektakuläre Fälle aufgeschreckt« sah (Lenckner FS Baumann 1992, 140, vgl. auch Götting NJW 2001, 585 zur BGH-Entscheidung ,Der Blaue Engel').

107 Vgl. auch E'62 (vor § 182) S.327 sowie AE-StGB-BT 1971, 29

108 Anders Peglau ZRP 1998, 250, der im Glauben an dessen »sozialpädagogische Funktion« den strafrechtlichen Schutz vor allem »aus Gründen der Bewußtseinsprägung « zum Einsatz bringen will, dagegen u.a. Kühl (s.o. Anm.15 S.6). 
nien der Analyse sind dabei - wie eingangs dargelegt - insbesondere die Kriterien der Funktionalität und Proportionalität.

\section{Regelungsstandort}

Der Einbau des vorgesehenen Straftatbestandes in den 15.Abschnitt des StGB-BT liegt zwar nahe, ob die Strafbarkeit mit einer abweichenden Konstruktion nicht auch im Nebenstrafrecht untergebracht werden könnte (soweit sie dort nicht ohnehin bereits hinreichend erfasst ist, s.o. III.3.), wäre aber immerhin erwägenswert. Innerhalb des 15.Abschnitts bietet sich - wie von den Entwürfen vorgesehen - eine Parallelkonstruktion zu $§ 201$ StGB an, weswegen die Einfügung als $§ 201$ a StGB ebenfalls nahe liegt. Zur Vermeidung von Wiederholungen sollte als Alternative allerdings in Betracht gezogen werden, die geplanten Tatbestandsvarianten in einen neu gefassten $\S 201$ StGB (Überschrift: Verletzung des persönlichen Lebensbereichs o.ä.) einzufügen, so wie es ansatzweise auch in $\S 146 \mathrm{AE}$ vorgesehen war, auf den die vorliegenden Entwürfe ja explizit Bezug nehmen. ${ }^{109}$

\section{Regelungsgehalt}

Im Folgenden wird der beabsichtigte Regelungsgehalt vor dem Hintergrund der zu schützenden Rechtsgüter analysiert in puncto Tatbestand (Tatsubjekt und -objekt, Tathandlung etc.) und Rechtswidrigkeit sowie weiterer für die Rechtsanwendung bedeutsamer Fragen (Versuch, Strafantrag). Gerade was diese tatbestandlichen und gesetzestechnischen Details betrifft, weisen die vorliegenden Entwürfe ihrerseits erhebliche Lücken auf, obwohl sich vieles gerade nicht ohne Weiteres aus § 201 StGB ergibt. $^{110}$

\section{a) Schutzgut}

Hinsichtlich des Schutzgutes des geplanten Tatbestandes herrscht zwischen den verschiedenen derzeit vorliegenden Entwürfen ein gewisses - zumindest terminologisches - Chaos $^{111}$ : Der E-FDP bezweckt den Schutz der Intimsphäre, so wie es unmissverständlich aus der Überschrift des Gesetzes als auch des geplanten § 201a hervorgeht, und so wie es dort zugleich als eigenständiges Tatbestandsmerkmal vorgesehen ist (in der Begründung ist z.T. auch vom persönlichen Lebens- und Geheimbereich die Rede, z.T. schlicht von Persönlichkeitsrechten); auch der E-Bayern stellt auf den Schutz der Intimsphäre ab, dies jedoch nur in den Überschriften, während im Tatbestand selbst vom höchstpersönlichen Lebensbereich die Rede ist (ebenso in der Begründung); der E-Union bezweckt hingegen laut Gesetzesüberschrift den Schutz der Privatsphäre, übersetzt dies aber in $§$ 201a selbst mit dem persönlichen Lebensbereich

109 AE-StGB-BT 1971, 32 ff.

110 Ebenso Francke (s.o. Anm.15) S.4 m.w.N. zur Uneinheitlichkeit des Regelungskomplexes der $\S 201 \mathrm{ff}$. StGB.

111 Das hat Tradition, vgl. bereits Kienapfel (s.o. Anm.4) S.18 ff. 
(ebenso in der Begründung, wo aber ebenfalls z.T. von Persönlichkeitsrechten die Rede ist).

Die Frage muss im Kontext des 15.Abschnitts gesehen werden, der Verletzungen des »persönlichen Lebens- und Geheimbereichs« erfasst. Während die §§ 202, 203, 204 und 206 StGB im Übrigen explizit auf »Geheimnisse« abstellen, will $§ 201$ die »Vertraulichkeit des Wortes ${ }^{112}$ vor Verletzungen schützen. Die Begriffe der Intimoder Privatsphäre sind dem Abschnitt jedoch fremd. ${ }^{113}$ Lässt sich der Begriff der Privatsphäre noch mit dem des persönlichen Lebens- und Geheimbereichs zur Deckung bringen ${ }^{114}$, hat die Intimsphäre bisher - jedenfalls als Tatbestandsmerkmal keinen Einzug in das Strafrecht erhalten (worauf auch E-FDP S.4 selbst hinweist). Das Bemühen, den Anwendungsbereich des geplanten § 201a StGB einzuschränken, indem der engere Begriff der »Intimsphäre« Verwendung findet (bzw. synonym der Begriff des »höchstpersönlichen Lebensbereichs « im E-Bayern), ist anzuerkennen und prinzipiell der Konstruktion des E-Union vorzuziehen, den durch den »persönlichen Lebensbereich« bewusst weiter gesteckten Anwendungsbereich erst durch eine Rechtswidrigkeitsklausel (in Abs.2) wieder einzugrenzen (s.u. h). Gleichwohl könnte sich der Begriff der »Intimsphäre « als strafrechtsdogmatischer Stolperstein in der Systematik des 15.Abschnitts erweisen und - auch im Hinblick auf Art.103 Abs.2 GG zu Auslegungsproblemen führen. ${ }^{115}$ Die weitergehende Einschränkung auf den Schutz (nur) »berechtigter Interessen« (E-FDP § 201a Abs. 3 S.1) - deren praktische Relevanz im Übrigen gering einzuschätzen ist (vgl. E-Bayern S.4, s.u. g) - dürfte die Probleme in der Rechtsanwendung einer Vorschrift, die ohnehin am Rande der Strafwürdigkeit angesiedelt ist, eher noch vertiefen und zusätzlich zu deren Marginalität beitragen (s.o. II.3.).

\section{b) Tat,subjekte' (Täter)}

Die Taten können im Grundtatbestand von jedermann begangen werden; lediglich für Amtsträger ist ein uneigentliches Sonderdelikt vorgesehen (wie in $§ 201$ Abs.3 StGB). Die Konstruktion erscheint sachgerecht: Eine Einschränkung des Täterkreises (wie in den $\S \S 203,204,206$ StGB) würde dem Schutzzweck nicht gerecht und ließe sich auch kaum formulieren. Wurde einem Dritten - jedoch unabhängig von dessen beruflicher oder sozialer Stellung - eine befugt hergestellte Aufnahme »anvertraut« (vgl.

112 Eigentlich die »Unbefangenheit«, vgl. NK-Jung § 201 Rn.2 m.w.N. sowie Schünemann ZStW 1978, 13, der die $\S \S 201,202$ StGB charakterisiert als »strafbares Eindringen in die mündliche und schriftliche Kommunikation «.

$113 \mathrm{Zu}$ den zivilrechtlichen Kategorien der Individual-, Privat- und Intimsphäre mit jeweils abgestuftem Schutzniveau vgl. Palandt-Thomas § 823 Rn.178 sowie Geis JZ 1991, 112 ff. und Richter NJW 1990, 2098 m.w.N.; grundlegend zur Intimsphäre in der Rechtsprechung etwa BGHZ 24, 72 und 73, 120 sowie NJW 1988, 1984, zum Strafrecht vgl. Krauß FS Gallas 1973, 367 ff., 381 einerseits und Schünemann ZStW 1978, 17 ff., 27 ff. andererseits.

114 So offenbar E-Union S.4, ähnlich Kühl (s.o. Anm.15 S.3).

115 Die Übertragung verfassungsrechtlicher Kriterien ins Strafrecht verläuft nicht so reibungslos, wie in E-FDP S.4 angenommen, ähnliche Bedenken bei Franke (s.o. Anm.15 S.6), Kühl (aaO S.3 f.) und Helgerth (aaO S.5), a.A. nur Hügel (aaO S.5). 
$\S 203$ Abs.1 StGB), die er sodann unbefugt offenbart, so soll lediglich nach $\S 201 \mathrm{a}$ Abs.1 Nr.3 E-Union eine Strafbarkeit in Betracht kommen (s.u.).

c) Tat,objekte‘ (Opfer)

»Angriffsobjekte « der in $\S 201 \mathrm{a}$ StGB vorgesehenen Tathandlungen sind einerseits andere Personen (die aufgenommen oder beobachtet werden), andererseits die Aufnahmen selbst, wobei es sich um unbefugte handeln kann (die gebraucht werden), aber auch um befugte (die missbraucht werden, was aber nur im E-Union vorgesehen ist, s.u. d).

Andere Personen können alle Menschen sein, unabhängig etwa vom Alter ${ }^{116}$ oder von ihrer etwaigen Prominenz, aber auch unabhängig davon, wo oder in welcher Situation sie sich befinden - eine tatbestandliche Eingrenzung soll lediglich stattfinden über das zusätzliche Merkmal der Verletzung der Intimsphäre bzw. des persönlichen Lebensbereichs (dazu unten e) und im E-FDP über deren Eignung zur Beeinträchtigung berechtigter Interessen (s.u. g). Lediglich der E-Bayern geht hier einen grundsätzlich anderen $\mathrm{Weg}^{117}$, indem nur solche Personen geschützt sind, die sich »in einer Wohnung oder einem gegen Einblick besonders geschützten Raum « befinden (wobei aber zusätzlich noch eine Verletzung ihres »höchstpersönlichen Lebensbereichs « verlangt wird). Dieser Vorschlag sieht sich zwar dem grundsätzlich begrüßenswerten Ziel verpflichtet, den Anwendungsbereich einzuschränken, um der »Gefahr [zu begegnen], das Übermaßverbot staatlichen Strafens sowie das strafrechtliche Bestimmtheitsgebot $\mathrm{zu}$ verletzen«, es drängt sich jedoch die Frage auf, ob damit einerseits ein Anwendungsbereich verbleibt, der die Einführung eines Straftatbestandes überhaupt noch »lohnt «, und ob er andererseits gerade jene Fallkonstellationen erfasst, die als »neue« Bedrohungen der Privatsphäre die Diskussion um die Einführung eines solchen Tatbestandes (wieder) entfacht haben (Einsatz von web-cams und spy-cams, Videoüberwachung etc.) - dazu passt es, dass ein spezifisches Amtsdelikt (s.o. b) im E-Bayern nicht vorgesehen ist, weil dessen Anwendungsbereich insoweit gegen Null tendieren dürfte. Eine dem Tatbestand des $§ 201$ StGB nachgebildete Konstruktion, dessen Eingrenzung über die »Nichtöffentlichkeit« (resp. Vertraulichkeit) des Wortes hergestellt wird, bietet sich für den geplanten $\S 201$ a StGB allerdings kaum an. ${ }^{118}$

»So hergestellte «, also unbefugte Aufnahmen sollen ihrerseits Tatobjekt sein, indem sie (in Anlehnung an $§ 201$ Abs.1 Nr.2 StGB) gebraucht oder anderen zugänglich

116 Nach Kühl (s.o. Anm.15 S.13) sollte der Schutzbereich auf Verstorbene erweitert werden.

117 Teilweise in Anlehnung an § 146 Abs.2 AE-StGB, ähnlich Peglau ZRP 1998, 251, der von Eingriffen »in den persönlichen Lebensbereich einer Person« spricht, »den diese erkennbar gegen die Kenntnisnahme durch Dritte abgeschirmt hat«, und Regelbeispiele vorschlägt, allerdings mit bedenklichen Weiterungen in Richtung eines allgemeineren Indiskretionsdelikts.

118 Ähnliche Bedenken bei Franke (s.o. Anm.15 S.4/5 und 8), der zu Recht darauf verweist, dass der BGH in seiner ersten ,Caroline von Monaco'-Entscheidung (BGHZ 133, 332) hervorgehoben hat, der Schutz der Privatsphäre beschränke sich gerade nicht auf den häuslichen Bereich (ebenso BVerfG NJW 2000, 1021, vgl. dazu Dörr JuS 2000, 912, krit. Ladeur ZUM 2000, 879 ff.), vgl. auch Kühl (s.o. Anm.15 S.18). 
gemacht werden (s.u. d), wobei diese Tatvariante nur dann eigenständige Bedeutung erlangen würde, wenn bereits ein Anderer die Aufnahme unbefugt hergestellt hat. Man mag hierin eine gewisse Vertiefung der bereits durch die Aufnahme herbeigeführten Persönlichkeitsrechtsverletzung, aber auch die eigentliche Rechtsgutsbeeinträchtigung sehen: so oder so erscheint die tatbestandliche Ergänzung adäquat. Ein Anwendungsproblem könnte darin liegen, dass unklar bleibt, worauf sich »so hergestellt« exakt bezieht: Nur auf die fehlende Befugnis (s.u. f) oder auch bereits auf die Verletzung der Intim- bzw. Privatsphäre? Sachgerecht dürfte nur eine Konstruktion sein, die sich auf Aufnahmen bezieht, deren Herstellung ihrerseits strafbar war. ${ }^{119}$

Einen qualitativen Sprung stellt die Erweiterung des Tatbestandes auf den Missbrauch befugt hergestellter Aufnahmen dar, wie er in $\S 201$ a Abs.1 Nr.3 E-Union vorgesehen ist (s.u. d), wobei völlig offen bleibt, was für Aufnahmen gemeint sind: Es könnte sich aber allenfalls um solche handeln, die den Betroffenen abbilden und wenigstens abstrakt geeignet sind, seine berechtigten Interessen zu beeinträchtigen.

\section{d) Tathandlungen}

Tathandlung soll einmal das (unbefugte) Aufnehmen einer anderen Person auf einen Bildträger sein (nur gemäß $§ 201$ a Abs.2 E-FDP auch deren Beobachtung, s.u.), zum Anderen das Gebrauchen oder Zugänglichmachen einer solchen Aufnahme.

In der Rechtsanwendung erscheint das Aufnehmen (= Herstellen einer Aufnahme, vgl. Nr.2; nicht aber das Kopieren oder Überspielen, s.u.) als Tathandlung zunächst unproblematisch, zumal weitgehend auf Rechtsprechung und Literatur zu § 201 Abs.1 Nr.1 StGB zurückgegriffen werden könnte. ${ }^{120}$ Die Probleme erweisen sich eher im Tatsächlichen, also beim Nachweis der Aufnahme, insbesondere wenn mittlerweile der Bildträger vernichtet oder die Aufnahme auf dem Bildträger gelöscht wurde - zur Minderung der Beweisprobleme auf die Versuchsstrafbarkeit zurückzugreifen (s.u. j), ist zwar pragmatisch gedacht, aber keine , saubere' Lösung.

Die Legaldefinition für den Bildträger ${ }^{121}$ lässt sich ohne Weiteres aus $\S 16$ Abs.2 UrhG übernehmen, wonach es sich um »Vorrichtungen zur wiederholbaren Wiedergabe von Bildfolgen « handeln muss, so dass als Bildträger auch solche »Vorrichtungen« gelten, die sich letztlich nur als Datenträger darstellen. Schwieriger ist die Frage, ob damit auch bereits die sog. »flüchtige Aufnahme « in den Arbeitsspeicher erfasst ist, die etwa nur zur unmittelbaren Bildübertragung (z.B. Videoüberwachung) dient, ohne gespeichert bzw. aufgezeichnet zu werden. ${ }^{122}$ Die in $\S 201 \mathrm{a}$ Abs.2 E-FDP enthaltene Tatbestandsvariante des Beobachtens (s.u.) deutet darauf hin, dass eine solche bloße Beobachtung »mit einem Bildaufnahmegerät oder anderen technischen Mitteln« noch keine Aufnahme auf einen Bildträger sein soll. Dies entspräche der o.g. Legaldefini-

119 Ähnlich zu § 201 Abs.1 Nr.2 StGB Kramer NJW 1990, 1762.

120 Nach Tröndle/Fischer $\S 201$ Rn.2 ist Aufnehmen die »unbefugte (heimliche) Verwandlung unmittelbarer Äußerungen in reproduzierbare Aufzeichnungen «.

121 Vgl. auch Tröndle/Fischer Rn.35 zu § 11 Abs.3 StGB; Kühl (s.o. Anm.15 S.7) stellt auf die Pepertuierung und jederzeitige Verwendbarkeit ab (ähnlich Lenckner FS Baumann 1992, 142). 
tion aus $\S 16$ Abs.2 UrhG, da der Arbeitsspeicher keine »wiederholbare« Wiedergabe der Bildfolgen ermöglicht. Die Beobachtung einer anderen Person etwa auf dem Display einer Digitalkamera wäre mithin noch keine Aufnahme auf einen Bildträger; sie könnte aber auch nicht als deren Versuch gewertet werden, solange dem Beobachter nicht nachgewiesen werden kann, dass die Speicherung beabsichtigt war (Tatentschluss).

Soll schon das (unbefugte) Beobachten einer Person von der Vorschrift erfasst werden, bedürfte es mithin eines gesonderten Tatbestandes, so wie er ausgerechnet in dem »liberalen « $\S 201 \mathrm{a}$ Abs.2 E-FDP vorgesehen ist (nicht hingegen im E-Union ${ }^{123}$ ). Ein solches Beobachten der unbefugten Aufnahme und deren Gebrauch gleichzustellen (»Ebenso wird bestraft ...«) versteht sich jedoch keinesfalls von selbst ${ }^{124}$ : Zwar kann auch das Beobachten (E-Bayern S.4: der »freche Blick«) - jedenfalls in bestimmten Situationen - einen Eingriff in die Privatsphäre bedeuten, und zwar unabhängig davon, ob es geheim oder offen geschieht, jedoch wurde gerade zur offenen polizeilichen Videoüberwachung herausgearbeitet, dass gegenüber der »bloßen« Beobachtung die Aufzeichnung (Aufnahme) den schwerwiegenderen Eingriff darstellt, insbesondere wegen der (nur) damit verbundenen Möglichkeiten der weiteren (insb. elektronischen) Verarbeitung, Distribution und Proliferation.

Dass die Strafbarkeit des Beobachtens beschränkt werden soll auf ein solches »mit einem Bildaufnahmegerät oder anderen technischen Mitteln $\ll^{125}$ leuchtet im Übrigen nur ein vor dem Hintergrund bereits angedeuteter Beweisprobleme: Damit sich der Mensch hinter der Kamera resp. am Monitor nicht damit herausreden kann, er habe gar nicht aufgenommen sondern »nur hingeschaut «, wird Letzteres kurzerhand in den Tatbestand einbezogen (und das noch dazu auch in der Versuchsvariante nach Abs.5,

122 Diese Frage ist nicht zu verwechseln mit der in § 11 Abs.3 StGB vorgesehenen Gleichstellung von Bild- und Datenträgern bei der Definition des spezifisch strafrechtlichen ,Schriften'-Begriffs, wobei auch Arbeitsspeicher als Datenspeicher behandelt werden (vgl. Tröndle/Fischer $\S 11$ Rn.36 sowie die aaO Rn.36a und $\S 184$ Rn.32 zu Recht geäußerte Kritik an der probl. Ausweitung des Datenspeicherbegriffs durch BGHSt 47, 55, dort in Bezug auf $\S \S 176 a$ Abs.2 iVm 184 Abs.3 StGB, vgl. auch Schmitz JA 1995, 480 f.).

123 Ungenau insofern E-Bayern, der ausdrücklich auch die Bild übertragung erfasst, ohne dass ersichtlich wird, ob es nur um die Übertragung bereits hergestellter Bildaufnahmen gehen soll (so der Wortlaut), oder auch um die bloße Übertragung zur Beobachtung ohne Aufzeichnung; insoweit ist auch die Begründung widersprüchlich, wenn es einerseits heißt, die »unbefugte Beobachtung « solle straffrei bleiben (S.4), andererseits aber auch sog. »Echtzeitübertragungen z.B. mittels sog. ,WebCams' oder ,SpyCams' ohne dauernde Speicherung der aufgenommenen [!?] Bilder « vom Tatbestand erfasst sein sollen (S.6); laut Hügel (s.o. Anm.15 S.8) sollen hierdurch gerade auch »Direktübertragungen« strafrechtlich erfasst werden, die noch keine »Aufnahmen $\ll$ sind.

124 Zwar weist § 201 Abs.2 S.1 Nr.1 StGB eine vergleichbare Konstruktion für das Abhören mittels Abhörgeräten auf, der Unterschied liegt jedoch darin, dass das Abhören nicht nur die Wahrnehmung des nichtöffentlich gesprochenen Wortes (und damit dessen technische Aufzeichnung) ermöglicht, sondern zugleich (und unabhängig von der technischen Aufzeichnung) dessen wörtliche oder sinngemäße Protokollierung, wie auch aus Nr.2 hervorgeht (vgl. zu dessen Einführung BT-Drs 11/6714 und Jung ZRP 1991, 169 sowie bereits BT-Drs 10/1618 S.10 und Schilling JZ 1980, 9 f.). 
s.u. j). Das macht aber spätestens dann keinen Sinn mehr, wenn das »technische Mittel « gar nicht geeignet war, Aufzeichnungen (Aufnahmen) herzustellen ${ }^{126}$ : So wie der Tatbestand in $\S 201 \mathrm{a}$ Abs.2 E-FDP formuliert ist, erfasst er auch den Einsatz eines banalen Fernglases - warum das strafbar sein soll, nicht aber das Hineinsehen in ein Schlafzimmerfenster mit bloßen Augen, leuchtet ebenso wenig ein. ${ }^{127}$ Schließlich bringt das Tatbestandsmerkmal des »Beobachtens « Rechtsanwendungs- und Beweisprobleme mit sich (ebenso E-Bayern S.5), denn Beobachten ist mehr als Sehen, so wie das Abhören in $§ 201$ Abs.2 S.1 Nr.1 StGB mehr ist als Hören. ${ }^{128}$ Es bedürfte deshalb im Einzelfall nicht nur Feststellungen dazu, dass überhaupt ein »Hinsehen « stattgefunden hat, sondern auch zu dessen Intensität und Dauer - kaum widerlegbare Schutzbehauptungen liegen nahe. ${ }^{129}$

Alles in Allem erscheint die Einbeziehung der Beobachtung (ohne gleichzeitige Aufzeichnung) in den Tatbestand nicht ratsam. Der um sich greifenden Videoüberwachung wäre damit jedenfalls nicht beizukommen. ${ }^{130}$ Soweit in diesem Kontext der Bedarf für eine strafrechtliche Absicherung gesehen wird (das eigentliche Problem ist, wie bereits erwähnt, die zunehmende Legalisierung der Überwachung und nicht deren Illegalität), sollte eine nebenstrafrechtliche Lösung im Datenschutzrecht geprüft werden (falls diese nicht bereits in $\S \S 44$ Abs.1, 43 Abs.2 Nr.1 iVm $\S 6$ b BDG besteht, s.o. III.3.b).

Auch der Gebrauch einer unbefugten Aufnahme oder deren Zugänglichmachen an Dritte bereitet zunächst einmal keine Anwendungsprobleme, wobei wiederum auf $\S 201$ Abs.1 StGB ${ }^{131}$ zurückgegriffen werden kann. Solche Probleme könnten sich aber ergeben hinsichtlich der weiteren Be- und Verarbeitung digitalisierter Bilddateien. Das Datenschutzrecht verwendet (z.B. in § 3 BDSG) den Begriff der automatisierten Verarbeitung, unterscheidet aber im Weiteren zwischen der Erhebung $(\S 3$

125 Mit einem Seitenblick auf $\S 201$ Abs.2 S.1 Nr.1 StGB sei ein weiteres Problem nur angedeutet: Umstritten ist, ob es bei den dort eingesetzten ,Abhörgeräten' nur um verbotene technische Einrichtungen geht (so BGH NJW 1982, 1398, vgl. Tröndle/Fischer § 201 Rn.7 m.w.N.), oder ob auch der Einsatz allgemein üblicher bzw. zugelassener Geräte tatbestandsmäßig sein kann (vgl. NK-Jung § 201 Rn.9 m.w.N.) - die Begründung in EFDP äußert sich dazu nicht explizit, weist allerdings darauf hin, die Formulierungen orientierten sich »wesentlich an denjenigen des § $201 \mathrm{StGB} \ll(\mathrm{S} .4)$, weshalb sich die o.g. Problematik auch in dem neuen $\S 201$ a StGB ergeben könnte.

126 Ähnlich Kühl (s.o. Anm.15 S.8).

127 Ebenso Hügel (s.o. Anm.15 S.9), die zu Recht auch darauf hinweist, dass jedem Aufnehmen ein Betrachten vorausgehe (aaO S.9,11, vgl. Kühl aaO S.8), so dass im E-FDP der Versuch des Abs.1 immer schon als Vollendung des Abs.2 strafbar wäre und die Strafbarkeit damit - so Helgerth (aaO S.6) - »in das Vorfeld ohnehin nicht eindeutig strafwürdiger Verhaltensweisen « ausgedehnt würde; eine solche, der Aufnahme vorgelagerte Beobachtung ist im Übrigen auch nicht vom Recht am eigenen Bild erfasst (Legler CR 1998, 439).

$128 \mathrm{Vgl}$. Wormer (s.o. Anm.58) S.199 ff.

129 Ebenso E-Bayern S.5, vgl. Schöch (s.o. Anm.15 S.4).

130 Soweit dies von der Verfassern des E-FDP überhaupt intendiert ist - die entsprechenden ,Befürchtungen“ von Helgerth (s.o. Anm.15 S.3 und 7) erscheinen allerdings überzogen.

131 Vgl. zum Tatbestandsmerkmal Gebrauchen z.B. auch die Kommentierungen zu $§ \S 267$ Abs.1, 268 Abs.1 Nr.1 oder 269 Abs.1 StGB. 
Abs.3 BDSG, entspricht hier quasi der Aufnahme), der Verarbeitung ( 33 Abs.4

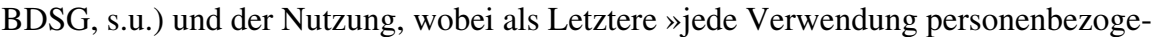
ner Daten [gilt], soweit es sich nicht um Verarbeitung handelt« ( 33 Abs.5 BDSG; vgl. auch § 3 Abs.2 DSG-NW). Setzte man im vorliegenden Kontext - was zunächst sprachlich nahe liegt - das Nutzen und Verwenden dem Gebrauch gleich, bliebe die Verarbeitung außen vor, also insbesondere das Speichern, Verändern, Übermitteln, Sperren und Löschen (vgl. § 3 Abs.4 BDSG; diff. § 3 Abs.2 S.1 DSG-NW). Während Sperren und Löschen hier weitgehend unberücksichtigt bleiben können ${ }^{132}$ und das Übermitteln auch mit dem Zugänglichmachen gleichgesetzt werden kann, bliebe die Frage, ob das Speichern oder Verändern ${ }^{133}$ einer Bilddatei als »Gebrauch« der Aufnahme subsumiert werden kann, die sich noch zuspitzt, wenn man sie auf die Tatbestandsvariante des Missbrauchs einer befugten Aufnahme ( $\$ 201 \mathrm{a}$ Abs.1 Nr.3 EUnion, s.u.) bezieht: Soll die Be- oder Verarbeitung einer befugt hergestellten Aufnahme gegen den Willen des Berechtigten bereits als deren »Gebrauch « strafbar sein (etwa noch im Versuchsstadium)? Damit dürfte wiederum der Bereich strafwürdigen Unrechts endgültig verlassen werden. Bliebe zu klären, ob bereits das bloße Betrachten der Aufnahme als deren Gebrauch einzustufen ist, was durch die Auslegung zu $\S 201$ Abs.1 Nr.2 StGB nahegelegt wird, wenn dort bereits »das Vorspielen vor sich selbst « als Gebrauch gewertet wird. ${ }^{134}$ Allerdings ist nicht ganz nachvollziehbar, wie die Rechtsgutsbeeinträchtigung der unbefugten Aufnahme allein dadurch gesteigert werden soll, dass sie betrachtet wird - selbst wenn man berücksichtigt, dass nicht der Aufnehmende selbst (der sich ja bereits nach Nr.1 strafbar gemacht hat) diese Tatbestandsvariante erfüllen kann, sondern nur ein Dritter (s.o. c).

Die Einbeziehung des Missbrauchs befugt hergestellter Aufnahmen in den Tatbestand ( $\$ 201 \mathrm{a}$ Abs.1 Nr.3 E-Union ${ }^{135}$ ) ist problematisch, weil sie den Anwendungsbereich noch einmal erheblich ausweitet: Damit wäre bereits unter Strafe gestellt, ein einzelnes verfängliches Urlaubsfoto, das von der berechtigten Person einer anderen Person zur Ansicht übergeben wurde, einem Dritten zu zeigen (gemäß Abs.4 reicht sogar der Versuch). Strafwürdiges Unrecht ist darin beim besten Willen nicht zu erkennen ${ }^{136}$, eher der Paradefall eines massenhaft begangenen Delikts, das allenfalls in

132 Nach NK-Jung § 201 Rn.7 soll allerdings auch das Überspielen einer (dadurch gelöschten) Tonaufnahme deren Gebrauch sein, was im Hinblick auf den Schutzzweck der Norm fraglich erscheint und eher in den Anwendungsbereich der $\S \S 303,303$ a StGB hineinspielt; soweit es im vorliegenden Kontext um die »Löschung, Unterdrückung, Unbrauchbarmachung oder Veränderung « einer Bilddatei (vgl. zu § 202a Abs.2 StGB oben Anm.59) gehen soll, bestünde jenseits des § 303a StGB allerdings überhaupt kein Regelungsbedarf.

133 Dazu Wanckel (s.o. Anm.46) S.183 f. m.w.N., insb. auch zu sog. »visuellen Lügen« (aaO S.184), insoweit überhaupt keine Bedenken bei Kühl (s.o. Anm.15 S.8).

134 Tröndle/Fischer § 201 Rn.6, ähnlich NK-Jung § 201 Rn.7.

135 Der insofern auch über § 201 StGB hinausgeht, vgl. NK-Jung § 201 Rn.8; die §§ 202a, 203 StGB weisen ebenfalls keine vergleichbare Konstruktion auf.

136 Ähnliche Bedenken bei Franke (s.o. Anm.15 S.8/9), nicht hingegen bei Kühl (aaO S.9 f.), diff. Hügel (aaO S.7), die dabei vorrangig an die Weitergabe einvernehmlich hergestellter Nacktfotos z.B. nach der Trennung denkt, was für sie »zweifelsohne strafwürdig« aber bereits durch $\S 33$ KUG erfasst sei - krit. dazu bereits Henkel DJT 42 D 93. 
ganz seltenen Ausnahmefällen (und dann einigermaßen willkürlich, s.o. II.3.) kriminalisiert würde. Anwendungsprobleme könnten sich darüber hinaus ergeben, weil unklar bleibt, ob die »andere Person«, deren persönlicher Lebensbereich verletzt sein soll, mit »der berechtigten Person«, gegen deren Willen die befugt hergestellte Aufnahme gebraucht wird, identisch sein muss. Das tatbestandsausschließende Einverständnis («gegen den Willen«, wobei es sich entgegen der Begründung - E-Union S.4 - nicht um eine rechtfertigende Einwilligung handelt) eröffnet schließlich ein weites Anwendungsfeld des vorsatzausschließenden Irrtums (s.u. f). Diese Tatbestandsvariante schießt weit über das Ziel hinaus und ist deshalb (zumindest in der vorliegenden Fassung) abzulehnen: »Es liegt im Risikobereich des Betroffenen, die Herstellung zu verhindern bzw. mit ihr nur eine verlässliche Person zu betrauen. Gegen die unbefugte Veröffentlichung (...) gewährt $§ 33$ KUG strafrechtlichen Schutz «. ${ }^{137}$

e) Verletzung der Intim- bzw. Privatsphäre

Alle vorliegenden Entwürfe grenzen den Anwendungsbereich der jeweiligen Tatbestände dadurch ein, dass sie neben den bereits erörterten (und für sich genommen nur abstrakt rechtsgutsgefährdenden) Tathandlungen eine konkrete Verletzung des Schutzguts fordern (s.o. a), während der E-FDP darüber hinaus noch eine spezielle tatbestandsausschließende Bagatellklausel vorsieht (s.u. g). Dabei ergeben sich lediglich graduelle Unterschiede, wenn einerseits eine Verletzung der Intimsphäre gefordert wird (E-FDP), andererseits eine solche des persönlichen (E-Union) oder sogar des höchstpersönlichen Lebensbereichs (E-Bayern).

Die Weite der Tatbestände (Ausnahme: E-Bayern, s.u.) erfordert in der Tat eine Einschränkung auf solche Taten, die das Schutzgut des 15.Abschnitts tangieren. Auch wenn die Konzeption es nahe legt, in den jeweiligen Tathandlungen regelmäßig eine entsprechende Schutzgutverletzung zu erkennen (praktische Indizwirkung), verlangen die vorgeschlagenen Regelungen («dadurch, dass ...«) doch in jedem Einzelfall einen über die Feststellung der Tathandlung hinausgehenden Nachweis einer solchen Verletzung, soll das Kriterium seiner Funktion gerecht werden, die Beliebigkeit des Tatbestandes im Hinblick auf den Schutz des »Lebens- und Geheimbereichs « einzufangen. In $\S 201$ StGB wird dies (neben dem punktuellen Tatbestandsausschluss in Abs.2 S.2, s.u. g) erreicht durch das Erfordernis der Nichtöffentlichkeit des aufgezeichneten oder abgehörten gesprochenen Wortes, die mit der Vertraulichkeit in der Überschrift korrespondiert - eine vergleichbare und im Hinblick auf Art.103 Abs.2 GG vorzugswürdige Konstruktion bietet sich für den geplanten $\S 201 \mathrm{a}$ StGB jedoch nicht an (s.o. c).

Trotz des enger gefassten Tatbestandes stellt auch der E-Bayern zusätzlich darauf $\mathrm{ab}$, dass durch die Tathandlung der »höchstpersönliche Lebensbereich« verletzt wird. Die Konstruktion leuchtet gerade auch im Vergleich zu § 201 StGB nicht auf Anhieb ein, verdeutlicht aber noch einmal das Bemühen, den Anwendungsbereich der Vorschrift stark einzuschränken.

137 So zu Recht E-Bayern S.7, a.A. Schöch (s.o. Anm.15 S.4 f.). 


\section{f) (Un)Befugnis}

Die Frage, ob die Herstellung der Aufnahme unbefugt war, ist nicht lediglich eine solche der Rechtswidrigkeit (so aber offenbar E-FDP S.4, s.u. h), sondern berührt jedenfalls dann bereits die Tatbestandsmäßigkeit, wenn es um das tatbestandsausschliessende Einverständnis der betroffenen Person geht. ${ }^{138}$

Die Aufnahme auf einen Bildträger erfolgt unbefugt, wenn dem Aufnehmenden eine entsprechende Befugnis fehlt - das hört sich trivialer an, als es ist, insbesondere wenn man sich die Konstruktion als Regel-Ausnahme-Verhältnis verdeutlicht: Die Regel ist zunächst einmal, dass der Aufnehmende keine Befugnis hat, ihm muss m.a.W. die Aufnahme nicht explizit untersagt worden sein, es reicht vielmehr, dass sie ihm von dem Berechtigten (in der Regel die verletzte Person selbst, möglicherweise auch deren gesetzliche Vertretung) nicht gestattet wurde. Da es sich um ein Tatbestandsmerkmal handelt, führt ein entsprechender Irrtum (also die irrtümliche Annahme eines Einverständnisses) zum Vorsatzausschluss und damit praktisch zur Straflosigkeit (was nicht zu verwechseln ist mit der sog. »mutmaßlichen Einwilligung «, die - wenn überhaupt als Rechtfertigungsgrund zu werten wäre, s.u. h).

Auch die »so hergestellte Aufnahme« (§ 201a Abs.1 Nr.2 E-FDP und E-Union) kann nur eine unbefugt hergestellte sein. ${ }^{139}$ Andererseits impliziert die vorgeschlagene Konstruktion, das Gebrauchen oder Zugänglichmachen der unbefugten Aufnahme müsse nicht seinerseits unbefugt sein - es kann jedoch keinem Zweifel unterliegen, dass auch das Einverständnis in Gebrauch oder Zugänglichmachung (einer zunächst unbefugt hergestellten Aufnahme) den Tatbestand entfallen lässt.

Das in der Tatbestandsvariante des Missbrauchs einer befugten Aufnahme ( $\$ 201 \mathrm{a}$ Abs.1 Nr.3 E-Union) zusätzlich aufgenommene Merkmal »gegen den Willen der berechtigten Person « tangiert ebenfalls die Tatbestandsmäßigkeit (unklar E-Union S.4, wo von Einwilligung die Rede ist) und stellt jenseits der Kategorie »unbefugt « nur klar, dass es nicht lediglich auf das fehlende Einverständnis ankommen soll, sondern auf einen explizit entgegenstehenden Willen des Berechtigten. ${ }^{140}$

Für die Tatbestandsvariante der Beobachtung ( $\$ 201 \mathrm{a}$ Abs.2 E-FDP) gilt hinsichtlich des Merkmals »unbefugt« schließlich nichts Anderes: Das Einverständnis der beobachteten Person schließt den Tatbestand, andere Befugnisgründe die Rechtswidrigkeit aus. In diesem Zusammenhang ist klarstellend noch einmal darauf hinzuweisen, dass keine der Tatbestandsvarianten eine heimliche Begehung voraussetzen, was auch nicht sachgerecht wäre: Das offene Aufnehmen oder Beobachten, Gebrauchen oder Zugänglichmachen wird nicht allein dadurch befugt, dass die verletzte Person

138 NK-Jung § 201 Rn.6 m.w.N., Wormer (s.o. Anm.58) S.154 ff., 224 ff., diff. Tröndle/ Fischer § 201 Rn.9 f., § 202 Rn.12 und § 203 Rn.31, vgl. auch Wölfl Jura 2000, 231, Schmitz JA 1995, 31 und grundlegend Krauß FS Gallas 1973, 384 sowie Lenckner FS Baumann 1992, $147 \mathrm{ff}$.

139 Das wird hier noch deutlicher als in $\S 201$ Abs.1 StGB, wo das Merkmal »unbefugt« vor die Nr.1 gezogen wurde, vgl. dazu NK-Jung § 201 Rn.8 und diff. Lenckner FS Baumann 1992, $145 \mathrm{ff}$.

140 Auch insofern ungenau E-Union S.4, genauer hingegen Kühl (s.o. Anm.15 S.10), der eine entsprechende Klarstellung ggfls. auch für $\S 201$ StGB vorschlägt. 
den Vorgang wahrnimmt, solange nicht aus der Duldung ersichtlich wird, dass sie damit einverstanden ist - andernfalls schließt ein entgegenstehender Wille die Befugnis aus (wobei sich freilich weitere Vorsatzprobleme ergeben können). ${ }^{141}$

\section{g) Tatbestandsausschluss}

In Anlehnung an $\S 201$ Abs.2 S.2 StGB ${ }^{142}$ geht E-FDP in der Tatbestandseingrenzung noch einen Schritt weiter und macht die Strafbarkeit davon abhängig, dass die Tat »geeignet ist, berechtigte Interessen der verletzten Person zu beeinträchtigen« (Abs.3 S.1). Das ist zwar gut gemeint und macht aus dem abstrakten Gefährdungsdelikt immerhin ein relativ konkretes (sog. ,Eignungsdelikt'), erscheint aber einigermaßen irrelevant: Dass eine bereits festgestellte Verletzung der Intimsphäre (s.o. e) nicht geeignet sein soll, berechtigte Interessen zu beeinträchtigen, ist praktisch kaum vorstellbar (ebenso E-Bayern S.4). Insofern überrascht auch die Charakterisierung als »Bagatellklausel« (E-FDP S.4), denn Verletzungen der Intimsphäre können schwerlich nur Bagatellen sein. Nach gängiger verfassungs- und zivilgerichtlicher Rechtsprechung ist die Intimsphäre im Übrigen absolut geschützt ${ }^{143}$, weshalb eine Bagatellklausel auch insofern irritiert. Letztlich erweist sich deren Übernahme aus $§ 201$ Abs.2 S.2 StGB als Fehlgriff. ${ }^{144}$

\section{h) Rechtswidrigkeit}

Die Frage der Rechtswidrigkeit stellt sich gewissermaßen auf zwei verschiedenen Ebenen: Einerseits sollen sich die Taten nur auf unbefugte Aufnahmen beziehen (Ausnahme: § 201a Abs.1 Nr.3 E-Union, s.o. d), andererseits ist in allen Entwürfen eine spezielle Rechtfertigungsregelung vorgesehen. Letzteres ist zweifellos Gegenstand der Rechtswidrigkeitsprüfung (s.u.), Ersteres hingegen nur, soweit es sich nicht bereits um einen Fall des tatbestandsausschließenden Einverständnisses handelt (s.o. f). Davon abgesehen handelt es sich nach h.M. lediglich um einen (eigentlich unnötigen ${ }^{145}$ ) expliziten Hinweis auf die allgemeine Rechtswidrigkeitsprüfung: Neben den »klassischen « Rechtfertigungsgründen, die allenfalls in Ausnahmefällen Anwendung finden dürften ${ }^{146}$, kommt einerseits die mutmaßliche Einwilligung in Betracht (die allerdings in den meisten Fällen daran scheitern wird, dass die Einwilligung des Berechtigten hätte eingeholt werden können), andererseits spezielle gesetzliche Befugnisnormen (insb. aus dem Polizei- und Strafprozessrecht) ... das muss hier nicht vertieft werden. ${ }^{147}$

141 Vgl. auch Schmitz JA 1995, 31.

142 Dazu Tröndle/Fischer $§ 201$ Rn.12 m.w.N.

143 Exempl. BGH NJW 1988, 1984 f. m.w.N., vgl. Loewenstein (s.o. Anm.72) § 18 Rn.27 sowie Delp (s.o. Anm.51 S.129 f.).

144 Ebenso Helgerth (s.o. Anm.15 S.6) sowie Franke (aaO S.7 und 9), der stattdessen eine dem § 86 Abs.3 StGB nachgebildete Adäquanzklausel oder eine Rechtswidrigkeitslösung à la $\S 240$ Abs.2 StGB vorschlägt, aber postwendend auf die auch damit verbundenen »Definitions- und Abgrenzungsprobleme « und »Tücken« verweist, abl. auch Kühl (aaO S.10 f.), der für eine teleologische Reduktion des Tatbestandes plädiert.

145 Diff. Klug FS Oehler S.401 f., der hierin den gesetzgeberischen Hinweis erblickt, dass Rechtfertigungen besonders nahe liegen können.

146 Diff. Kühl (s.o. Anm.15 S.11 f.) für § 34 StGB im Hinblick auf die Presse. 
Die Tathandlungen sollen darüber hinaus auch dann nicht rechtswidrig sein, wenn sie entweder »zur Ausführung oder Verteidigung von Rechten oder zur Wahrnehmung berechtigter Interessen « begangen wurden ( $\$ 201 \mathrm{a}$ Abs.2 E-Union) oder - hier beschränkt auf den Gebrauch und das Zugänglichmachen - »zur Wahrnehmung überragender öffentlicher Interessen« ( $\$ 201 \mathrm{a}$ Abs.3 S.2 E-FDP). Letztere Klausel ist an $\S 201$ Abs.2 S.3 StGB angelehnt (s.u.), Erstere eher an $§ 193$ StGB: Da die Wahrnehmung berechtigter Interessen nur von einer Mindermeinung als allg. Rechtfertigungsgrund anerkannt wird ${ }^{148}$, wäre es richtig, ihn hier gesondert zu erwähnen - wenn es denn überhaupt gerechtfertigt ist, ihn hier zur Anwendung zu bringen: In der Begründung beider Vorschläge heißt es (insofern überraschend) einhellig, aus Art.5 Abs.1 GG könne sich ein Rechtfertigungsgrund ergeben, »wenn die Bedeutung der Information für die Unterrichtung der Öffentlichkeit und Meinungsbildung die Nachteile des Rechtsbruchs überwiegt«, wobei auf »erhebliche Missstände« abgestellt wird (E-FDP S.4 und E-Union S.4); das Argument ist nachvollziehbar, aber zu eng gefasst. Zwar erscheint es auf den ersten Blick systematisch angezeigt, die Klausel des $§ 201$ Abs.2 S.3 StGB auch auf den geplanten $§ 201$ a StGB zu übertragen, sie bezieht sich dort aber ausschließlich auf die öffentliche Mitteilung, weswegen auch lediglich auf überragende »öffentliche « Interessen abgestellt wird. ${ }^{149}$ Im Hinblick auf die Aufnahme selbst und deren Gebrauch ist die Bezugnahme auf berechtigte Interessen i.S.d. § 193 StGB sachgerechter, weil sich »auch aus anderen Lebenssachverhalten rechtfertigende Umstände ergeben « können. ${ }^{150}$

Freilich, das sei abschließend noch einmal hervorgehoben (s.o. II.2.), sind gerade die vielfältigen Möglichkeiten der Rechtfertigung (bzw. der Tatbestandseingrenzung, s.o.) die wunden Punkte des strafrechtlichen Schutzes des persönlichen Lebens- und Geheimbereichs ( $\S \S 201$ ff. StGB), die an der Sinnhaftigkeit der Konstruktion zweifeln lassen - repräsentieren sie doch zugleich Zweifel an der Rechtmäßigkeit einer Kriminalisierung der weitaus meisten Fälle.

\section{j) Versuchsstrafbarkeit}

Alle vorliegenden Entwürfe sehen - offenbar in Anlehnung an $§ 201$ Abs.4 StGB ${ }^{151}$ eine Versuchsstrafbarkeit vor (E-FDP: Abs.5, E-Union: Abs.4, E-Bayern: Abs.3),

147 Überblick bei Tröndle/Fischer § 201 Rn.11 und Wölfl Jura 2000, 231 ff. m.w.N., exempl. auch Becker/Smeets Jura 2000, 354 und Schmitz JA 1995, 31; zur Frage der Notwehr gegen unbefugtes Fotografieren vgl. u.a. OLG Düsseldorf NJW 1994, 1971 m.w.N., zu den Grenzen der Notwehr gegen ,Spanner" hingegen BayObLG NJW 1962, 1782 (dazu Erdsiek NJW 1962, 2242 einerseits sowie Rötelmann MDR 1964, 207 f. und Schultz MDR 1981, 198 f. andererseits, vgl. auch Schünemann JuS 1979, 275 ff.).

148 Vgl. z.B. LK-Jähnke § 203 Rn.82, anders Schmitz JA 1995, 31, Tenckhoff JuS 1989, 198 und Wölfl Jura 2000, 234 m.w.N.

$149 \mathrm{Vgl}$. auch E-Bayern S.7, der stattdessen - seinerseits nicht recht nachvollziehbar - ganz auf eine entsprechende Klausel verzichtet; vgl. im Übrigen Tröndle/Fischer $\S 201$ Rn.13 und die Kritik von Lenckner FS Baumann 1992, $151 \mathrm{f}$.

150 So zu Recht E-Union S.4, ähnlich Kühl (s.o. Anm.15 S.12), a.A. AE-StGB-BT S.35; zur Frage einer etwaigen Rechtfertigung durch sog. ,Beweisnotstand“ vgl. Kramer NJW 1990, 1762 f. m.w.N. 
ohne deren Notwendigkeit jedoch zu begründen (auch insoweit herrscht leider Einigkeit). Ein allgemeines strafgesetzgeberisches Prinzip bei der Pönalisierung des Versuchs ist (jenseits $\S 23$ Abs.1 Alt.1 StGB, der auf die Schwere der Strafandrohung abstellt) nicht zu erkennen. Trotz der gemäß $§ 23$ Abs.2 StGB möglichen Strafmilderung stellt die Versuchsstrafbarkeit eine legitimationsbedürftige Verlagerung des strafrechtlichen Zugriffs in das Vorfeld einer Rechtsgutsverletzung dar, wo also gewissermaßen (jedenfalls dem »Opfer«) noch »gar nichts passiert « ist. Umso niedriger die Strafandrohung und umso weiter der Straftatbestand selbst (hier als Gefährdungsdelikt) im diesem Vorfeld angesiedelt ist, umso fragwürdiger wird die darüber noch hinausgehende Kriminalisierung des Versuchs. ${ }^{152}$

Vor diesem Hintergrund ist die vorgesehene Versuchsstrafbarkeit alles Andere als selbstverständlich $^{153}$ und eigentlich nur zu erklären unter Verweis auf $\S 201$ Abs.4 StGB einerseits und die bereits erwähnten Beweisschwierigkeiten andererseits - beides ist aber nicht geeignet, sie hinreichend zu legitimieren. So bleibt insbesondere unberücksichtigt, dass die Versuchsstrafbarkeit des § 201 StGB die Ausnahme im 15.Abschnitt darstellt, denn bei allen anderen Delikten wurde darauf mit guten Gründen verzichtet. Die Strafbarkeit des bloßen Ansetzens zur Bildaufnahme oder deren Gebrauch bzw. Zugänglichmachung oder gar lediglich zur Beobachtung im Einzelfall damit legitimieren zu wollen, dass generelle Beweisschwierigkeiten beseitigt werden müssten ${ }^{154}$, sollte als Verstoß gegen die Regeln der strafgesetzgeberischen »Kunst« zurückgewiesen werden.

\section{k) Antragserfordernis (aber kein Privatklagedelikt)}

Abgesehen von den Amtsdelikten (soweit vorgesehen, s.o. b) soll es sich durchweg um absolute Antragsdelikte handeln, indem $\S 201 \mathrm{a}$ StGB in den $\S 205$ StGB einbezogen wird. ${ }^{155}$ Das ist zwar stimmig, macht aber zugleich deutlich, dass öffentliches Interesse an der Kriminalisierung solcher »Taten « grundsätzlich nicht existiert (vgl. II. 2.), entsprechende Verfahren also ausschließlich im »privaten « Interesse der Antragsberechtigten betrieben würden ${ }^{156} \ldots$ weswegen nur schwer nachvollziehbar ist, dass es sich nicht zugleich um Privatklagedelikte handeln soll: Im Katalog des $§ 374$ Abs.1 StPO wurde $\$ 201$ StGB ausgespart, weswegen dies offenbar auch für den geplanten $\S 201$ a StGB nicht erwogen wurde (soweit man überhaupt daran gedacht hat). Der

151 Nach Tröndle/Fischer $\S 201$ Rn.16 stellt die Inbetriebnahme von Abhör- oder Aufzeichnungsgeräten den Beginn der Versuchsstrafbarkeit dar, während deren Installation noch (straflose) Vorbereitungshandlung und Vollendung erst beim Hören oder Aufzeichnen erreicht ist, ähnlich Kühl (s.o. Anm.15 S.8).

152 Umgekehrte Kritik an $\S 202$ a StGB bei Ernst NJW 2003, 3237.

153 So aber offenbar Kühl (s.o. Anm.15 S.8), anders Schöch (aaO S.5).

154 So aber bereits AE-StGB-BT S.35, um dem »Einwand des Täters zu begegnen, das eingebaute Gerät habe nicht funktioniert und keine Bilder übertragen«.

155 Dazu Schmitz JA 1995, 31 f.; zu § 33 KUG vgl. Mestmäcker/Schulze (s.o. Anm.72) § 33 Rn.2/3.

156 Denen aber gleichwohl das öffentliche Strafverfahrensrecht mitsamt seinen Akteuren (insb. Polizei) und Zwangsmitteln (insb. Beschlagnahme etc.) zur Instrumentatlisierung anheimgestellt wird (s.o. II.3.). 
Sache nach wäre es allerdings ebenso gut vertretbar wie für den $\S 202$ StGB und den $\S 33$ KUG (\$ 374 Abs.1 Nr.3 und 8 StPO). ${ }^{157}$ Würde entgegen der vorliegenden Entwürfe auch die Einbeziehung des geplanten $\S 201 \mathrm{a}$ StGB in den Katalog vorgesehen, wäre freilich zu überlegen, zumindest auch den bereits bestehenden $§ 201$ StGB mit aufzunehmen.

\section{Sanktionsregelung}

Die vorgesehenen Rechtsfolgen orientieren sich an $\S 201$ StGB (und entsprechen $\S 202 \mathrm{a} \mathrm{StGB}$ ) und sind deshalb wohl zu vertreten. Gleichwohl sollte - auch in einer Gesamtbetrachtung der Strafrahmen des 15. Abschnitts - noch einmal bedacht werden, dass die $\S \S 202$ und 203 nur eine Freiheitsstrafe bis zu einem Jahr vorsehen (ebenso $\S 33 \mathrm{KUG}^{158}$ ): Worin eigentlich die Unterschiede in der gesetzgeberischen Schwere-Einschätzung des Unrechts begründet liegen sollen, bleibt unerfindlich. So oder so dürfte die Verhängung von Freiheitsstrafen (auch im Hinblick auf $\S \S 46$ a und 47 Abs.1 StGB) ohnehin die absolute Ausnahme bleiben. ${ }^{159}$

Die Übernahme der Einziehungsregelung aus § 201 Abs.5 StGB in den geplanten $\S 201$ a StGB wäre stimmig, wobei die im E-Bayern vorgesehene Formulierung ${ }^{160}$ den Vorzug verdiente. Bei der Anwendung müsste angesichts der Geringfügigkeit der Delikte in der Ausübung des Ermessens allerdings der Grundsatz der VerhältnismäBigkeit besondere Beachtung finden, was umso mehr gilt für die korrespondierenden strafprozessualen Sofortmaßnahmen der Beschlagnahme gemäß $§ 111$ b,c StPO.

\section{Resumee}

Insgesamt überwiegt - neben der Detailkritik an den vorgelegten Entwürfen - die Skepsis: Mag auch die Legitimität des verfolgten Schutzzwecks unbestritten sein, erscheint doch die Funktionalität der geplanten Regelungen mehr als fraglich (von gesetzestechnischen Mängeln und strafrechtsdogmatischen Widersprüchlichkeiten ganz abgesehen), zumal die Gebote der Subsidiarität und Proportionalität des Strafrechtseinsatzes nicht durchgängig gewahrt werden. Ungeachtet dessen sollte vor einer weiteren punktuellen Lückenfüllung eine Gesamtrevision des strafrechtlichen Schutzes des Lebens- und Geheimbereichs im 15.Abschnitt des StGB-BT erwogen werden.

157 Ausf. zu § 33 KUG Helle (s.o. Anm.69) S.225 m.w.N.; ebenso Hügel (s.o. Anm.15 S.12), a.A. Schöch (aaO S.5), der vielmehr dafür plädiert, auch § $33 \mathrm{KUG}$ aus dem $\S 374 \mathrm{StPO}$ zu streichen.

158 Im E-Bayern ist sowohl für § 201a StGB als auch für den (zu ändernden) § 33 KUG eine Freiheitsstrafe von bis zu zwei Jahren vorgesehen.

159 Selbst im Falle einer Strafschärfung für Amtsdelikte (s.o. 2.b); Kühl (s.o. Anm.15 S.12) fordert darüber hinaus sogar eine Strafschärfung für gewerbsmäßige Taten, was MedienMit- und Zuarbeiter zusätzlich verunsichern dürfte.

160 Ebenso bereits der E-BaWü (BR-Drs.164/03 vom 11.3.2003 in $\S 201$ a Abs.6), der sich allerdings auch nur bezüglich dieser Marginalie vom E-FDP unterschied. 